\title{
DEFORMATION CONDITIONS FOR PSEUDOREPRESENTATIONS
}

\author{
PRESTON WAKE ${ }^{1}$ and CARL WANG-ERICKSON ${ }^{2}$ \\ ${ }^{1}$ Institute for Advanced Study, Princeton, NJ 08540, USA; \\ email: pwake@ias.edu \\ ${ }^{2}$ Department of Mathematics, Imperial College London, London SW7 2AZ, UK; \\ email: c.wang-erickson@imperial.ac.uk
}

Received 5 September 2017; accepted 17 June 2019

\begin{abstract}
Given a property of representations satisfying a basic stability condition, Ramakrishna developed a variant of Mazur's Galois deformation theory for representations with that property. We introduce an axiomatic definition of pseudorepresentations with such a property. Among other things, we show that pseudorepresentations with a property enjoy a good deformation theory, generalizing Ramakrishna's theory to pseudorepresentations.
\end{abstract}

2010 Mathematics Subject Classification: 11F70 (primary); 16R99, 11S20 (secondary)

\section{Introduction}

The deformation theory of Galois representations has been used extensively to realize and study the proposed Langlands correspondence between Galois representations and automorphic representations. Depending on the setting in which the correspondence is being studied, one wants to set up the deformation theory so that it parameterizes exactly the Galois representations with certain properties. A collection of such properties is known as a 'deformation condition' or 'deformation datum'. Ramakrishna [Ram93] proved that for any deformation condition $\mathcal{C}$ satisfying a basic stability property, the deformation problem for $\mathcal{C}$ is representable relative to the unrestricted deformation problem.

In applications, and especially when working with residually reducible Galois representations, Galois pseudorepresentations are often more accessible 
than Galois representations. A Galois pseudorepresentation is the data of a characteristic polynomial for each element of the Galois group, satisfying appropriate compatibility conditions. However, it has not been clear how to apply deformation conditions-which apply to Galois modules-to pseudorepresentations.

The point of this paper is to solve this problem by axiomatically translating deformation conditions for representations into deformation conditions for pseudorepresentations. In particular, we construct universal deformation rings for pseudorepresentations satisfying such a condition. Also, it follows from our techniques that a deformation condition cuts out a closed locus in any family of Galois representations, generalizing the case of local families considered in [Ram93]. We expect that the various constructions we make, especially the universal Cayley-Hamilton algebra defined by a condition, will find many applications.

We were stimulated to develop this theory for application in the companion paper [WWE17b] (see also [WWE18b]). After this work was complete, the second-named author identified Galois cohomological data that controls the deformation theory of these pseudodeformation rings: see [WE18b], especially [WE18b, Theorem 3.4.1].

1.1. Background. To give a more thorough explanation of our results, we define our scope. Fix a prime $p$ and a profinite group $G$. We assume that $G$ satisfies the finiteness condition $\Phi_{p}$ (see Section 1.4). We are interested in studying the categories $\mathcal{R e p}_{G}$ of representations of $G$ and $\operatorname{PsR}_{G}$ of pseudorepresentations of $G$, and the related moduli spaces $\operatorname{Rep}_{G}$ and $\operatorname{PsR}_{G}$.

Let $\mathcal{C}$ be a condition on finite-cardinality $\mathbb{Z}_{p}[G]$-modules such that $\mathcal{C}$ is closed under isomorphisms, subquotients, and finite direct sums (we call such a condition stable). Ramakrishna [Ram93] showed that the formal deformation functor of representations with $\mathcal{C}$ is representable.

The goal of this paper is to sensibly extend the condition $\mathcal{C}$ so that it applies to pseudorepresentations. One difficulty in doing this is that pseudorepresentations are defined as collections of functions; they need not come in any obvious way from a $\mathbb{Z}_{p}[G]$-module.

The main tool we use to bridge the gap between $G$-pseudorepresentations and $G$-modules is the category $\mathcal{C H}_{G}$ of Cayley-Hamilton representations of $G$, which was introduced by Chenevier [Che14], building on work of BellaïcheChenevier [BC09], and further developed by second-named author [WE18a]. The data of a Cayley-Hamilton representation of $G$ includes a $\mathbb{Z}_{p}[G]$-module, so it is possible to relate these objects to condition $\mathcal{C}$. Moreover, $\mathcal{C} \mathcal{H}_{G}$ is closely related to both $\mathcal{R e p}_{G}$ and $\mathrm{PsR}_{G}$; the key properties can be summarized as follows: 
- $\mathcal{C H}_{G}$ broadens the category of representations of $G$, in the sense that there is an inclusion $\mathcal{R e p}_{G} \hookrightarrow \mathcal{C H} \mathcal{H}_{G}$ and an induced-pseudorepresentation functor $\psi: \mathcal{C H}_{G} \rightarrow$ PsR $_{G}$ extending the usual functor $\mathcal{R e p}_{G} \rightarrow \operatorname{PsR}_{G}$.

- $\psi$ is essentially surjective, which is not true for $\left.\psi\right|_{\mathcal{R e p}_{G}}$.

- $\mathcal{C H}_{G}$ has a universal object, the 'universal Cayley-Hamilton representation' of $G$, which we denote by $E_{G}$.

- $E_{G}$ is finitely generated as a module over its base ring.

The first two conditions say that Cayley-Hamilton representations generalize the notation of representations to an extent sufficient to see all pseudorepresentations. The last two conditions mean that $\mathcal{C} \mathcal{H}_{G}$ is still manageable in size; in particular, it reduces constructions to finite-cardinality $\mathbb{Z}_{p}[G]$-modules, so one can apply the condition $\mathcal{C}$. We review the theory of Cayley-Hamilton representations in Section 2.2.

1.2. Results. We use the above properties of $\mathcal{C H}_{G}$ to give the definition of a Cayley-Hamilton representation of $G$ with condition $\mathcal{C}$ (Definition 2.5.2). The Cayley-Hamilton representations of $G$ with condition $\mathcal{C}$ form a full subcategory $\mathcal{C H}_{G}^{\mathcal{C}} \subset \mathcal{C H}_{G}$. We say that a pseudorepresentation has condition $\mathcal{C}$ if there is a Cayley-Hamilton representation that induces it. More precisely, we define the category of pseudorepresentations satisfying condition $\mathcal{C}$, denoted by $\operatorname{PsR}_{G}^{\mathcal{C}}$, as the essential image $\operatorname{PsR}_{G}^{\mathcal{C}} \subset \operatorname{PsR}_{G}$ of $\psi$ restricted to $\mathcal{C} \mathcal{H}_{G}^{\mathcal{C}} \subset \mathcal{C} \mathcal{H}_{G}$ (Definition 2.5.4).

We view these new definitions as being the heart of our paper. Our main results are meant to justify why we think this notion is a useful generalization of Ramakrishna's theory. We show that it has the same geometric and universality properties as Ramakrishna's, and that it specializes to his definition in cases where both definitions apply. Importantly, we also give tools for checking whether certain pseudorepresentations (including many of the ones that come up naturally) have this property. In some cases, we are also able to give tools for computing these objects in terms of natural Selmer-type groups.

Our main general results are as follows.

(1) The category $\mathcal{C} \mathcal{H}_{G}^{\mathcal{C}}$ has a universal object $E_{G}^{\mathcal{C}}$, which is a quotient of the universal object $E_{G}$ in $\mathcal{C H}_{G}$ (Theorem 2.5.3).

(2) We define the notion of a faithful Cayley-Hamilton $G$-module $M$ (it is a $G$ module with extra structures giving rise to a Cayley-Hamilton representation $E_{M}$ of $G$ ). We prove that $M$ satisfies $\mathcal{C}$ if and only if $E_{M}$ satisfies $\mathcal{C}$ (Theorem 2.6.4).

(3) Property $\mathcal{C}$ is a Zariski-closed condition on both $\operatorname{Rep}_{G}$ and $\operatorname{PsR}_{G}$ (Corollary 2.7.1). In particular, for a fixed residual pseudorepresentation, there 
is a pseudodeformation-with- $\mathcal{C}$ ring that is a quotient of the universal pseudodeformation ring (Theorem 2.5.5).

Many of the pseudorepresentations that arise naturally in the study of modularity lifting come from faithful Cayley-Hamilton $G$-modules (see Example 2.6.3), and result (2) is very useful for showing that such a pseudorepresentation has $\mathcal{C}$. Result (3) is a generalization of a theorem of Ramakrishna [Ram93, Proposition 1.2] for residually irreducible representations, and, in that case, we prove that our construction agrees with his (Corollary 3.3.5).

In Sections 3-4, we restrict our attention to the subcategories of $\mathcal{C H}_{G}, \mathcal{R e p}_{G}$, and $\mathrm{PsR}_{G}$ that are residually multiplicity-free (see Definition 3.2.1). For the sake of this introduction, we abuse notation and use the same letters for the residually multiplicity-free versions. With this restriction, it is known that $E_{G}$ admits the extra structure of a generalized matrix algebra, a theory developed by BellaïcheChenevier (see [BC09, Section 1.5] and [WWE17a, Section 4.1]). Using this extra structure, we prove the following results in Sections 3 and 4, respectively:

(4) The quotient $E_{G}^{\mathcal{C}}$ of $E_{G}$ is uniquely characterized in terms of $\mathcal{R e p}_{G}^{\mathcal{C}}$ (Theorem 3.3.1).

(5) The structure of $E_{G}^{\mathcal{C}}$ is related to extension groups with condition $\mathcal{C}$ (Theorem 4.3.5).

We view this last result as especially important, as it allows one to compute with some of our constructions. In particular, it allows one to give a kind of upper bound on the size of the pseudodeformation ring in terms of Selmer-type groups, and this is useful in proving modularity-lifting theorems (see Remark 4.0.1). This method is used in our papers [WWE17b, WWE18b].

REMARK 1.2.1. In the body of the paper, we mostly discuss variants of these results for the versions of the categories that have a fixed residual pseudorepresentation $\bar{D}$. To translate into the form stated in this introduction, one can apply the results of Section 2.2, particularly Theorem 2.2.5.

1.3. Context. We are motivated by the case that $G$ is a quotient of an absolute Galois group of a global field and $\mathcal{C}=\left\{\mathcal{C}_{v}\right\}$ is a condition on the restrictions of representations of $G$ to its decomposition groups $G_{v}$, especially those conditions $\mathcal{C}_{v}$ that arise from $p$-adic Hodge theory when $v \mid p$. We discuss the examples we have in mind in Section 5.

The unavailability of a notion of pseudorepresentations satisfying said conditions has been an obstacle to generalizing the use of the deformation 
theory of Galois representations to the residually reducible case. In particular, in the papers [WWE17b, WWE18b], we require the particular condition $\mathcal{C}_{v}$ that a $\mathbb{Z}_{p}\left[G_{v}\right]$-module arises as the generic fiber of a finite-flat group scheme over the ring of integers of a $p$-adic local field. (This is the same condition that especially motivated [Ram93].)

In our previous work [WWE18a, WWE17a], we considered the ordinary condition on two-dimensional global Galois representations (see also [CS19] and [WE18a, Section 7]). However, the ordinary condition is of a rather different flavor, as it does not apply readily to a finite-cardinality $\mathbb{Z}_{p}[G]$-module without extra structure.

1.4. Notation and conventions. Rings $R$ are commutative and Noetherian, unless otherwise noted. Algebras are associative but not necessarily commutative, and are usually finitely generated. For an algebra $E$, the term ' $E$-module' is used to mean 'left $E$-module', unless otherwise stated, and we let $\operatorname{Mod}_{E}$ denote the category of left $E$-modules.

When $A$ is a local ring, to say that $B$ is commutative local $A$-algebra means that $B$ is a local ring equipped with a morphism of local rings $A \rightarrow B$.

We study integral $p$-adic representations and pseudorepresentations of a profinite group $G$, which is assumed to satisfy the $\Phi_{p}$ finiteness condition of Mazur [Maz89]: the maximal pro- $p$ quotient of every finite index subgroup of $G$ is topologically finitely generated.

We work with categories of topological rings $R$ discussed in Section 2.2, giving finitely generated $R$-modules a natural topology. Algebras $E$ over $R$ are also understood to have their natural topology, either being finitely generated as $R$ modules or of the form $R[G]$. Actions of $G$ and homomorphisms $G \rightarrow E^{\times}$are understood to be continuous. Pseudorepresentations $D: E \rightarrow R$ (respectively $D: G \rightarrow R$ ) are also understood to be continuous, which means that the characteristic polynomial coefficient functions $E \rightarrow R$ (respectively $G \rightarrow R$ ) defined in Definition 2.1.1(8) are continuous.

1.4.1. A note on attribution We wish to make it clear that the notions of pseudorepresentation, Cayley-Hamilton algebra, and generalized matrix algebra, which are used in an essential way in this paper, are due to Chenevier [Che14] and Bellaïche-Chenevier [BC09, Ch. 1], building on works of others including Wiles [Wil88], Procesi [Pro87], Taylor [Tay91], Nyssen [Nys96], and Rouquier [Rou96].

Where possible, we have attempted to cite the original sources directly. In some cases, we also cite [WE18a] for background on these objects because the category of profinite-topological Cayley-Hamilton representations of a profinite group was 
developed there, adapting the nontopological version of [Che14, Section 1.22]. In contrast, we note that Chenevier developed the profinite-topological notion of pseudorepresentation [Che14, Section 3], which we rely on without making any additional contributions.

\section{Deformation conditions for Cayley-Hamilton representations}

In this section, we develop the theory of Cayley-Hamilton representations with a deformation condition $\mathcal{C}$. The first three subsections are mainly review. In Section 2.1, we begin by recalling from [Che14] and [WE18a, Section 2] the nontopological theory of pseudorepresentations and Cayley-Hamilton algebras, their compatible representations, and moduli spaces of these (see also [WWE18a] for further background and examples). In Section 2.2, we review the modifications of this theory for topological groups. In Section 2.3, we discuss the stable conditions of Ramakrishna [Ram93], and show that there is a well-defined 'maximal quotient with $\mathcal{C}$ of a finite-cardinality $\mathbb{Z}_{p}[G]$-module.

The main new constructions are given in Section 2.4. There, we show that the 'maximal quotient with $\mathcal{C}$ ' of the universal Cayley-Hamilton algebra retains its algebra structure. We also show that, for an algebra quotient of a CayleyHamilton algebra, there is a maximal further quotient that is Cayley-Hamilton. Combining these constructions, we obtain a 'maximal Cayley-Hamilton quotient with $\mathcal{C}$ ' of the universal Cayley-Hamilton algebra.

The main results and definitions of the section are stated and proven in Section 2.5. We define the category of Cayley-Hamilton representations with $\mathcal{C}$, and show that the object constructed in Section 2.4 is a universal object in this category. We define a pseudorepresentation with $\mathcal{C}$ as a pseudorepresentation that comes from a Cayley-Hamilton representations with $\mathcal{C}$, and prove that the corresponding deformation functor is prorepresentable.

In Section 2.6, we introduce the notion of Cayley-Hamilton $G$-module. Many pseudorepresentations that arise from modular forms (and more generally, automorphic forms) come from Cayley-Hamilton $G$-modules (see Example 2.6.3). We prove a criterion for when such pseudorepresentations have $\mathcal{C}$. In Section 2.7, we apply this criterion to prove a generalization of Ramakrishna's theorem from formal families of representations to more general families.

2.1. Pseudorepresentations, compatible representations, and CayleyHamilton algebras. Our starting point is following definition, due to Chenevier [Che14].

DEFINITIONS 2.1.1. Let $R$ be a ring, let $E$ be an $R$-algebra, and let $\mathcal{G}$ a group. 
(1) A polynomial law is a natural transformation $D: E \otimes_{R}(-) \rightarrow(-)$ of set-valued functors on commutative $R$-algebras, where (-) is the forgetful functor from $R$-algebras to sets. It is multiplicative if, for each commutative $R$-algebra $A$, the function $D_{A}: E \otimes_{R} A \rightarrow A$ satisfies $D_{A}(1)=1, D_{A}(x y)=$ $D_{A}(x) D_{A}(y)$ for all $x, y \in E \otimes_{R} A$. It has degree $d$ if, for each commutative $R$-algebra $A$, we have $D_{A}(b x)=b^{d} D_{A}(x)$ for all $x \in E \otimes_{R} A$ and all $b \in A$.

(2) A pseudorepresentation, denoted $D: E \rightarrow R$ or $(E, D)$, is a multiplicative polynomial law of degree $d$ from $E$ to $R$, for some $d \geqslant 1$. We call $d$ the dimension of $D$, and $R$ the scalar ring of $(E, D)$. The data of $D$ consists of a function $E \otimes_{R} A \rightarrow A$ for each commutative $R$-algebra $A$, which we denote by $D_{A}$.

(3) A pseudorepresentation of $\mathcal{G}$ with coefficients in $R$, denoted $D: \mathcal{G} \rightarrow R$, is a pseudorepresentation of $R[\mathcal{G}]$.

(4) If $D: E \rightarrow R$ is a pseudorepresentation, and $x \in E$, we define the characteristic polynomial $\chi_{D}(x, t) \in R[t]$ by $\chi_{D}(x, t)=D_{R[t]}(t-x)$. It is monic of degree equal to the dimension $d$ of $D$. We define the trace $\operatorname{Tr}_{D}(x)$ to be the additive inverse of the coefficient of $t^{d-1}$ in $\chi_{D}(x, t)$.

REMARK 2.1.2. This notion of pseudorepresentation is called a 'determinant' by Chenevier. The notion of 'pseudorepresentation' of a group was first considered by Wiles [Wil88, Lemma 2.2.3] in the case $d=2$, and by Taylor [Tay91, Section 1] in general. Taylor's definition was also considered by Rouquier [Rou96], who considered $R$-algebras (not just groups), and called the resulting objects 'pseudo-caractères' (or 'pseudocharacters', in English). By [Che14, Lemma 1.12], $\operatorname{Tr}_{D}: E \rightarrow R$ is a 'pseudocharacter' in the sense of Taylor and Rouquier. By [Che14, Proposition 1.29], the map $D \mapsto \operatorname{Tr}_{D}$ is a bijection if $(2 d) ! \in R^{\times}$.

EXAMPLE 2.1.3. There is a $d$-dimensional pseudorepresentation det $: M_{d}(R) \rightarrow$ $R$ defined by letting $\operatorname{det}_{A}: M_{d}(A) \rightarrow A$ be the determinant for all commutative $R$-algebras $A$. More generally, if $E$ is an Azumaya $R$-algebra of degree $d$, for example $V$ is a projective $R$-module of rank $d$ and $E=\operatorname{End}_{R}(V)$, then there is a $d$-dimensional pseudorepresentation det $: E \rightarrow R$ given by the reduced norm on $E$.

EXAMPLE 2.1.4. If $V$ is a projective $R$-module of rank $d$ and $\rho: R[\mathcal{G}] \rightarrow$ $\operatorname{End}_{R}(V)$ is a representation of $\mathcal{G}$, then there is an associated pseudorepresentation $\psi(\rho): \mathcal{G} \rightarrow R$. It is defined, for a commutative $R$-algebra $A$ and $x \in A[\mathcal{G}$ ], by

$$
\psi(\rho)_{A}(x)=\operatorname{det}_{A}\left(\left(\rho \otimes_{R} A\right)(x)\right),
$$

where det is as in the previous example. 
Informally, $\psi(\rho)$ is the composition of $\rho$ and det. In the following definition, we make this 'composition' construction explicit, and introduce notation for some other standard ways to produce new pseudorepresentations from old ones.

Definitions 2.1.5. Let $f: R \rightarrow R^{\prime}$ be a ring homomorphism, let $E$ and $E^{\prime}$ be an $R$-algebra and an $R^{\prime}$-algebra, respectively, and let $g: E \otimes_{R} R^{\prime} \rightarrow E^{\prime}$ be a morphism of $R^{\prime}$-algebras. Let $\mathcal{G}$ be a group.

(1) Let $D: E \rightarrow R$ be a pseudorepresentation. The base change of $D$ by $f$, denoted $f \circ D: E \otimes_{R} R^{\prime} \rightarrow R^{\prime}$, is the pseudorepresentation of $E \otimes_{R} R^{\prime}$ defined by $(f \circ D)_{A}(x)=D_{A}(x)$, where $A$ is a commutative $R^{\prime}$-algebra, and $x \in E \otimes_{R} A=\left(E \otimes_{R} R^{\prime}\right) \otimes_{R^{\prime}} A$.

When $f$ is understood, we write $D \otimes_{R} R^{\prime}$ instead of $f \circ D$.

(2) Let $D^{\prime}: E^{\prime} \rightarrow R^{\prime}$ be a pseudorepresentation. The composition of $D^{\prime}$ and $g$, denoted $D^{\prime} \circ g: E \otimes_{R} R^{\prime} \rightarrow R^{\prime}$, is the pseudorepresentation of $E \otimes_{R} R^{\prime}$ defined by $\left(D^{\prime} \circ g\right)_{A}(x)=D_{A}^{\prime}\left(\left(g \otimes_{R^{\prime}} A\right)(x)\right)$, where $A$ is a commutative $R^{\prime}$-algebra and $x \in E \otimes_{R^{\prime}} A$.

(3) A morphism of pseudorepresentations $\rho:(E, D) \rightarrow\left(E^{\prime}, D^{\prime}\right)$ is the data of a pair $(f, g)$, such that $f \circ D=D^{\prime} \circ g$. We define $\psi(\rho)=f \circ D=D^{\prime} \circ g$.

A morphism of pseudorepresentations of $\mathcal{G}$ is a morphism of pseudorepresentations $(R[\mathcal{G}], D) \rightarrow\left(R^{\prime}[\mathcal{G}], D \otimes_{R} R^{\prime}\right)$ where the homomorphism $R^{\prime}[\mathcal{G}] \rightarrow R^{\prime}[\mathcal{G}]$ is the identity.

(4) If $D: E \rightarrow R$ is a pseudorepresentation and $\rho: \mathcal{G} \rightarrow E^{\times}$is a homomorphism, there is an induced homomorphism $\tilde{\rho}: R[\mathcal{G}] \rightarrow E$, which defines a morphism of pseudorepresentations $(R[\mathcal{G}], D \circ \tilde{\rho}) \rightarrow(E, D)$. We abuse notation and denote this morphism by $\rho$, and write $\psi(\rho): \mathcal{G} \rightarrow R$ for the resulting pseudorepresentation of $\mathcal{G}$.

It is not, in general, true that any pseudorepresentation $D: \mathcal{G} \rightarrow R$ is of the form $\psi(\rho)$ for some representation $\rho$ of $\mathcal{G}$ as in Example 2.1.4. It is, of course, true that $D=\psi(\rho)$ for some morphism $\rho$ of pseudorepresentations $(R[\mathcal{G}]$, $D \circ \tilde{\rho}) \rightarrow(E, D)$ as in Definition 2.1.5(4) (we could take $E=R[\mathcal{G}]$ and $\tilde{\rho}$ to be the identity). However, it is a surprising fact that the analogous statement is true if we restrict $(E, D)$ to be in the more restrictive class of Cayley-Hamilton pseudorepresentations [Che14, Section 1.17]: see Corollary 2.2.12.

Definition 2.1.6. We call a pseudorepresentation $D: E \rightarrow R$ CayleyHamilton when $E$ is finitely generated as an $R$-algebra, and, for every 
commutative $R$-algebra $A$ and every $x \in E \otimes_{R} A$, the element $x$ satisfies the characteristic polynomial $\chi_{D}(x, t) \in A[t]$ associated to it by $D$. That is, $D$ is Cayley-Hamilton when $\chi_{D}(x, x)=0$ for all $x \in E \otimes_{R} A$. When $D: E \rightarrow R$ is Cayley-Hamilton, we call the pair $(E, D)$ a Cayley-Hamilton R-algebra.

An important property of Cayley-Hamilton algebras is the following finiteness result.

Proposition 2.1.7. If $(E, D)$ is a Cayley-Hamilton R-algebra, then $E$ is finitely generated as an $R$-module.

Proof. This follows from [WE18a, Proposition 2.13], which uses the theory of PIalgebras. Indeed, $E$ is finitely generated as an $R$-algebra (by definition of CayleyHamilton algebra) and $R$ is Noetherian by convention.

Of course, the pseudorepresentation $\left(M_{d}(R)\right.$, det) of Example 2.1.3 is a Cayley-Hamilton algebra (by the Cayley-Hamilton Theorem), and we think of Cayley-Hamilton representations as generalizations of this example. Just as algebra homomorphisms $E \rightarrow M_{d}(R)$ are given the special name representation, we follow Chenevier and give certain morphisms of pseudorepresentations the special name Cayley-Hamilton representation.

DEFINITIONS 2.1.8. Let $\mathcal{G}$ be a group.

(1) A Cayley-Hamilton representation of a pseudorepresentation $(E, D)$ is a morphism of pseudorepresentations $\rho:(E, D) \rightarrow\left(E^{\prime}, D^{\prime}\right)$ with $\left(E^{\prime}, D^{\prime}\right)$ a Cayley-Hamilton algebra.

(2) A Cayley-Hamilton representation of $\mathcal{G}$, denoted $\left(E^{\prime}, \rho, D^{\prime}\right)$, is a morphism of pseudorepresentations $(R[\mathcal{G}], D) \rightarrow\left(E^{\prime}, D^{\prime}\right)$ such that the map $R[\mathcal{G}] \rightarrow$ $E^{\prime}$ comes from a homomorphism $\rho: \mathcal{G} \rightarrow E^{\prime \times}$.

(3) If $(E, D)$ is also Cayley-Hamilton, we also refer to a Cayley-Hamilton representation $(E, D) \rightarrow\left(E^{\prime}, D^{\prime}\right)$ as a morphism of Cayley-Hamilton algebras.

(4) A morphism of Cayley-Hamilton representations of $\mathcal{G}$, written $(E, \rho, D) \rightarrow$ $\left(E^{\prime}, \rho^{\prime}, D^{\prime}\right)$, is a morphism of Cayley-Hamilton algebras $(E, D) \rightarrow\left(E^{\prime}, D^{\prime}\right)$ such that $\rho^{\prime}=\left(E \rightarrow E^{\prime}\right) \circ \rho$.

We let $\mathcal{C H}_{\mathcal{G}}$ denote the category of Cayley-Hamilton representations of $\mathcal{G}$, with morphisms as defined in (3). 
EXAMPLE 2.1.9. Let $(E, D)$ be a pseudorepresentation over $R$, and let $V$ be a projective $A$-module of rank $d$ for a commutative $R$-algebra $A$. Let $\left(\operatorname{End}_{A}(V)\right.$, det) be the pseudorepresentation defined in Example 2.1.3, which is a CayleyHamilton algebra. A Cayley-Hamilton representation $(E, D) \rightarrow\left(\operatorname{End}_{A}(V)\right.$, det $)$ is the data of a representation $\rho: E \rightarrow \operatorname{End}_{A}(V)$ of $E$ that is compatible with the pseudorepresentation, that is, it satisfies $D=\operatorname{det} \circ \rho$.

This example motivates the following (perhaps more familiar) definition.

Definition 2.1.10. Let $D: E \rightarrow R$ be a $d$-dimensional pseudorepresentation. Let $A$ be a commutative $R$-algebra.

(1) A framed compatible representation of $(E, D)$ over $A$ is an $R$-algebra homomorphism $\rho_{A}: E \rightarrow M_{d}(A)$ such that $D \otimes_{R} A=\operatorname{det} \circ \rho_{A}$. We denote by $\operatorname{Rep}_{E, D}^{\square}$ the set-valued functor which assigns to a commutative $R$-algebra $A$ the set of all framed compatible representations of $(E, D)$ over $A$.

(2) A compatible representation of $(E, D)$ over $A$ is a pair $\left(V_{A}, \rho_{A}\right)$, where $V_{A}$ is a projective rank $d A$-module and $\rho_{A}: E \rightarrow \operatorname{End}_{A}(V)$ is an $R$-algebra homomorphism such that $D \otimes_{R} A=\operatorname{det} \circ \rho_{A}$. We denote by $\operatorname{Rep}_{E, D}$ the Spec $R$-groupoid (indeed, a stack) which assigns to a commutative $R$-algebra $A$ a category whose objects are the compatible representations of $(E, D)$ over $A$ and whose morphisms are isomorphisms of such data.

REMARK 2.1.11. The functor $\operatorname{Rep}_{E, D}^{\square}$ and the groupoid $\operatorname{Rep}_{E, D}$ are representable over Spec $R$; see for example Theorem 2.2.11. In particular, the natural adjoint action of $\mathrm{GL}_{d}$ on the affine Spec $R$-scheme $\operatorname{Rep}_{E, D}^{\square}$ provides a smooth presentation for $\operatorname{Rep}_{E, D}$ as a Spec $R$-algebraic stack.

Note that, by Example 2.1.9, compatible representations are a special kind of Cayley-Hamilton representation. We view Cayley-Hamilton representations as a natural generalization of compatible representations.

2.2. Representations of a profinite group. For the remainder of the section, we fix a profinite group $G$ that satisfies the $\Phi_{p}$ condition. Recall the conventions from Section 1.4 regarding continuity.

Let $\mathrm{Adm}_{\mathbb{Z}_{p}}$ denote the category of admissible topological (not necessarily Noetherian) rings where $p$ is topologically nilpotent (see [Gro60, Definition 0.7.1.2, page 60] for the definition of admissible). We let $\operatorname{Tfg}_{\mathbb{Z}_{p}} \subset \operatorname{Adm}_{\mathbb{Z}_{p}}$ be the full subcategory of topologically finitely generated objects (those $A \in \operatorname{Adm}_{\mathbb{Z}_{p}}$ for which there exists a (nontopological) homomorphism $\mathbb{Z}_{p}\left[x_{1}, \ldots, x_{n}\right] \rightarrow A$ 
with dense image), which are Noetherian rings. For $R \in \operatorname{Tfg}_{\mathbb{Z}_{p}}$, we define $\operatorname{Tfg}_{R}$ as the slice category of $\operatorname{Tfg}_{\mathbb{Z}_{p}}$ over $R$. We often use the following fact.

LemmA 2.2.1. Let $R \in \mathrm{Tfg}_{\mathbb{Z}_{p}}$ and let $A \in \mathrm{Tfg}_{R}$. For any $x \in A$ nonzero, there exists an commutative local $R$-algebra of finite cardinality and an $R$-algebra homomorphism $f: A \rightarrow B$ such that $f(x) \neq 0$. In particular, a surjection $g$ : $A \rightarrow A^{\prime}$ in $\operatorname{Tfg}_{R}$ is determined by the natural transformation $\operatorname{Hom}_{R-\mathrm{alg}}\left(A^{\prime},-\right) \rightarrow$ $\operatorname{Hom}_{R-\mathrm{alg}}(A,-)$ of functors on commutative local $R$-algebras of finite cardinality.

Proof. We leave this as an exercise. The main point is that, for any ideal of definition $I$ for $A$, the ring $A / I^{n}$ is a finitely generated $\mathbb{Z}$-algebra, and consequently a Noetherian Jacobson ring.

DEFINITION 2.2.2. For $A \in \mathrm{Tfg}_{\mathbb{Z}_{p}}$, a representation of $G$ with coefficients in $A$ is a finitely generated projective $A$-module $V_{A}$ of constant rank with an $A$-linear $G$-action $\rho_{A}$. We write $\operatorname{Rep}_{G}$ for the category of representations, fibered in groupoids over $\operatorname{Tfg}_{\mathbb{Z}_{p}}$ via the forgetful functor $\left(V_{A}, \rho_{A}\right) \mapsto A$. We write $\operatorname{Rep}_{G}^{\square}$ for the category defined just as $\operatorname{Rep}_{G}$, but with the additional data of an $A$-basis for $V_{A}$.

Write $\operatorname{PsR}_{G}$ for the category of pseudorepresentations of $G$, fibered in groupoids over $\operatorname{Tfg}_{\mathbb{Z}_{p}}$.

We write $\psi$ for the functor $\psi: \operatorname{Rep}_{G} \rightarrow \operatorname{PsR}_{G}$ over $\operatorname{Tfg}_{\mathbb{Z}_{p}}$ that sends a representation $\rho_{A}$ to its induced pseudorepresentation $\psi\left(\rho_{A}\right)$.

We decompose each of these categories by dimension $d \geqslant 1$, writing $\operatorname{Rep}_{G}^{d} \subset$ $\operatorname{Rep}_{G}$, and so forth.

To understand $\operatorname{PsR}_{G}^{d}$, we introduce deformations. Given a finite field $\mathbb{F} / \mathbb{F}_{p}$, we let $\hat{\mathcal{C}}_{W(\mathbb{F})} \subset \operatorname{Tfg}_{\mathbb{Z}_{p}}$ be the category of complete Noetherian commutative local $W(\mathbb{F})$-algebras $\left(A, \mathfrak{m}_{A}\right)$ with residue field $\mathbb{F}$.

DEFINITION 2.2.3. Let $\bar{D}: G \rightarrow \mathbb{F}$ be a pseudorepresentation. Its deformation functor $\operatorname{PsDef}_{\bar{D}}: \hat{\mathcal{C}}_{W(\mathbb{F})} \rightarrow$ Sets is

$$
A \mapsto\left\{D: A[G] \rightarrow A \text { such that } D \otimes_{A} \mathbb{F} \simeq \bar{D}\right\}
$$

and elements of $\operatorname{PsDef}_{\bar{D}}(A)$ are called deformations of $\bar{D}$ or pseudodeformations.

Remarkably, when one varies $\bar{D}$ over a certain set of finite field-valued pseudorepresentations known as residual pseudorepresentations (see [WE18a, Definition 3.4] for the definition), $\operatorname{PsDef}_{\bar{D}}$ captures all of $\operatorname{PsR}_{G}^{d}$, in the following sense. 
THEOREM 2.2.5 (Chenevier). Assume G satisfies $\Phi_{p}$.

(1) Given a finite field $\mathbb{F}$ and a pseudorepresentation $\bar{D}: G \rightarrow \mathbb{F}$, the functor $\operatorname{PsDef}_{\bar{D}}$ is represented by an object $\left(R_{\bar{D}}, \mathfrak{m}_{\bar{D}}\right) \in \hat{\mathcal{C}}_{W(\mathbb{F})}$.

(2) There is an isomorphism

$$
\operatorname{PsR}_{G}^{d} \cong \coprod_{\bar{D}} \operatorname{Spf} R_{\bar{D}}
$$

where $\bar{D}$ varies over d-dimensional residual pseudorepresentations.

Proof. See [Che14, Propositions 3.3, 3.7 and Corollary 3.14].

Let $\operatorname{Rep}_{\bar{D}}$ (respectively $\operatorname{Rep}_{\bar{D}}^{\square}$ ) denote the fiber in $\operatorname{Rep}_{G}$ (respectively $\operatorname{Rep}_{G}^{\square}$ ) of $\psi$ over $\operatorname{Spf} R_{\bar{D}}$, where $\bar{D}$ is a residual pseudorepresentation, so that we have

$$
\operatorname{Rep}_{G}^{d} \cong \coprod_{\bar{D}} \operatorname{Rep}_{\bar{D}}, \quad \operatorname{Rep}_{G}^{\square, d} \cong \coprod_{\bar{D}} \operatorname{Rep}_{\bar{D}}^{\square},
$$

where $\bar{D}$ varies over $d$-dimensional residual pseudorepresentations.

Now, and for the rest of this section, we fix a finite field $\mathbb{F} / \mathbb{F}_{p}$ and a pseudorepresentation $\bar{D}: G \rightarrow \mathbb{F}$. In light of the decompositions (2.2.6) and (2.2.7), we lose no scope in our study of $p$-adic families by fixing this choice.

Definition 2.2.8. Let $A \in \operatorname{Tfg}_{\mathbb{Z}_{p}}$. We say that a pseudorepresentation $D: G \rightarrow$ $A$ has residual pseudorepresentation $\bar{D}$ when $\operatorname{Spf} A \rightarrow \operatorname{PsR}_{G}^{d}$ is concentrated over Spf $R_{\bar{D}}$. We write $D_{\bar{D}}^{u}: G \rightarrow R_{\bar{D}}$ for the universal pseudodeformation of $\bar{D}$.

A Cayley-Hamilton representation $\left(E, \rho: G \rightarrow E^{\times}, D: E \rightarrow A\right)$ of $G$ over $A \in \operatorname{Tfg}_{\mathbb{Z}_{p}}$ has residual pseudorepresentation $\bar{D}$ if its induced pseudorepresentation $\psi(\rho): G \rightarrow A$ has residual pseudorepresentation $\bar{D}$. In particular, a representation $\left(V_{A}, \rho_{A}\right) \in \operatorname{Rep}_{G}^{d}(A)$ has residual pseudorepresentation $\bar{D}$ when $\psi\left(\rho_{A}\right)$ does.

We let $\mathcal{C H}_{G, \bar{D}}$ denote the full subcategory of $\mathcal{C H}_{G}$ (introduced in Section 1.1) whose objects have residual pseudorepresentation $\bar{D}$. The natural transformation

$$
\operatorname{Rep}_{\bar{D}} \rightarrow \mathcal{C H}_{G, \bar{D}}
$$

arises from the Cayley-Hamilton representation structure on a representation of Example 2.1.4. This natural transformation commutes with the inducedpseudorepresentation functor $\psi$. 
We observe that $\operatorname{Rep}_{\bar{D}}$ parameterizes representations of $G$ with residual pseudorepresentation $\bar{D}$. From [WE18a, Theorem 3.8] we know that $\operatorname{Rep}_{\bar{D}}$ and $\operatorname{Rep}_{\bar{D}}^{\square}$ admit natural algebraic models over $\operatorname{Spec} R_{\bar{D}}$. By this we mean that there exists a finite type affine scheme (respectively algebraic stack) over Spec $R_{\bar{D}}$ whose $\mathfrak{m}_{\bar{D}}$-adic completion is $\operatorname{Rep}_{\bar{D}}^{\square}$ (respectively $\operatorname{Rep}_{\bar{D}}$ ). This implies that $\operatorname{Rep}_{\bar{D}}^{\square}$ is an affine formal scheme and $\operatorname{Rep}_{\bar{D}}$ is a formal algebraic stack, both formally of finite type over $\operatorname{Spf} R_{\bar{D}}$.

This algebraic model arises from a canonical universal Cayley-Hamilton representation of $G$ with residual pseudorepresentation $\bar{D}$, which we now define.

THEOREM 2.2.10. The category $\mathcal{C} \mathcal{H}_{G, \bar{D}}$ has a universal object $\left(E_{\bar{D}}, \rho^{u}: G \rightarrow\right.$ $\left.E_{\bar{D}}^{\times}, D_{E_{\bar{D}}}^{u}: E_{\bar{D}} \rightarrow R_{\bar{D}}\right)$. In particular, $E_{\bar{D}}$ is a finitely generated $R_{\bar{D}}$-module. The map $\rho^{u}: R_{\bar{D}}[G] \rightarrow E_{\bar{D}}$ is surjective and $D_{E_{\bar{D}}}^{u}: E_{\bar{D}} \rightarrow R_{\bar{D}}$ is a factorization of the universal pseudodeformation $D_{\bar{D}}^{u}: G \rightarrow R_{\bar{D}}$ through $E_{\bar{D}}$.

Proof. See [WE18a, Proposition 3.6].

Another way of stating the final sentence of Theorem 2.2.10 is that the induced pseudorepresentation $\psi\left(\rho^{u}\right):=D_{E_{\bar{D}}}^{u} \circ \rho^{u}: G \rightarrow R_{\bar{D}}$ of the universal CayleyHamilton representation $\rho^{u}$ (with residual pseudorepresentation $\bar{D}$ ) is equal to the universal deformation $D_{\bar{D}}^{u}: G \rightarrow R_{\bar{D}}$ of $\bar{D}$.

The following theorem is proved using (2.2.9) and the universal property of $\rho^{u}$.

THEOREM 2.2.11 [WE18a, Theorem 3.7]. There is an isomorphism of topologically finite type formal algebraic stacks (respectively formal schemes) on $\operatorname{Tfg}_{\mathbb{Z}_{p}}$,

$$
\operatorname{Rep}_{\bar{D}} \stackrel{\sim}{\longrightarrow} \operatorname{Rep}_{E_{\bar{D}}, D_{E_{\bar{D}}}^{u}}, \quad \operatorname{Rep}_{\bar{D}}^{\square} \stackrel{\sim}{\longrightarrow} \operatorname{Rep}_{E_{\bar{D}}, D_{\bar{E}_{\bar{D}}}^{u}}^{\square} .
$$

Corollary 2.2.12. Let $A \in \mathrm{Tfg}_{\mathbb{Z}_{p}}$. Let $D: G \rightarrow$ A be a pseudorepresentation. Then there exists a Cayley-Hamilton algebra over A that induces it.

Proof. By Theorem 2.2.5, we reduce to the case that $D$ has a fixed residual pseudorepresentation and deduce that there exists a homomorphism $R_{\bar{D}} \rightarrow A$. Then the Cayley-Hamilton representation

$$
\left(E_{\bar{D}} \otimes_{R_{\bar{D}}} A, \rho^{u} \otimes_{R_{\bar{D}}} A: G \rightarrow E_{\bar{D}} \otimes_{R_{\bar{D}}} A, D_{E_{\bar{D}}}^{u} \otimes_{R_{\bar{D}}} A\right)
$$

of $G$ over $A$ induces $D$. 
2.3. Stability. Let $\operatorname{Mod}_{\mathbb{Z}_{p}[G]}^{\text {in }} \subset \operatorname{Mod}_{\mathbb{Z}_{p}[G]}$ be the full subcategory whose objects have finite cardinality.

Definition 2.3.1. A condition on $\operatorname{Mod}_{\mathbb{Z}_{p}[G]}^{\text {fin }}$ is a full subcategory $\mathcal{C} \subset \operatorname{Mod}_{\mathbb{Z}_{p}[G]}^{\text {in }}$. We say an object of $\operatorname{Mod}_{\mathbb{Z}_{p}[G]}^{\text {in }}$ satisfies condition $\mathcal{C}$ or has $\mathcal{C}$ if the object is in $\mathcal{C}$.

A condition $\mathcal{C}$ is stable if it is preserved under isomorphisms, subquotients, and finite direct sums in $\operatorname{Mod}_{\mathbb{Z}_{p}[G]}^{\text {fin }}$. In other words, $\mathcal{C}$ is stable if

(1) for every object $A$ in $\mathcal{C}$ and all isomorphisms $f: A \rightarrow B$ in $\operatorname{Mod}_{\mathbb{Z}_{p}[G]}^{\text {in }}$, the object $B$ is also in $\mathcal{C}$, and

(2) for every object $A$ in $\mathcal{C}$ and all morphisms $f: A \rightarrow B$ and $g: C \rightarrow A$ in $\operatorname{Mod}_{\mathbb{Z}_{p}[G]}^{\text {fin }}$, the kernel of $f$ and cokernel of $g$ are in $\mathcal{C}$, and

(3) for every finite collection of objects $A_{1}, \ldots, A_{n}$ of $\mathcal{C}$, the direct sum $A_{1} \oplus$ $\cdots \oplus A_{n}$ in $\operatorname{Mod}_{\mathbb{Z}_{p}[G]}^{\mathrm{fin}}$ is an object of $\mathcal{C}$.

EXAMPLE 2.3.2. Let $H \subset G$ be a normal subgroup. Let $\mathcal{C} \subset \operatorname{Mod}_{\mathbb{Z}_{p}[G]}^{\text {fin }}$ be the full subcategory of objects where the $G$ action factors through the quotient $G \rightarrow$ $G / H$. Then $\mathcal{C}$ is stable (as $\mathcal{C} \cong \operatorname{Mod}_{\mathbb{Z}_{p}[G / H]}^{\text {fin }}$ ).

EXAMPLE 2.3.3. Let $H_{1}, \ldots, H_{n} \subset G$ be subgroups, and, for $i=1, \ldots, n$, let $\mathcal{C}_{i} \subset \operatorname{Mod}_{\mathbb{Z}_{p}\left[H_{i}\right]}^{\text {fin }}$ be a condition. Then there is a condition $\mathcal{C} \subset \operatorname{Mod}_{\mathbb{Z}_{p}[G]}^{\mathrm{fin}}$ defined by the Cartesian square

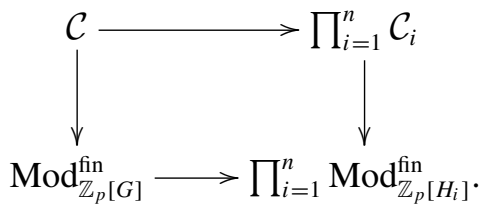

In other words, an object $M \in \operatorname{Mod}_{\mathbb{Z}_{p}[G]}^{\text {fin }}$ has $\mathcal{C}$ if and only if the restriction $\left.M\right|_{H_{i}} \in$ $\operatorname{Mod}_{\mathbb{Z}_{p}\left[H_{i}\right]}^{\text {fin }}$ has $\mathcal{C}_{i}$ for all $i=1, \ldots, r$. If all $\mathcal{C}_{i}$ are stable, then this $\mathcal{C}$ is stable.

For examples of conditions $\mathcal{C}$ that are of use in arithmetic, see Section 5. For the rest of this section, we fix a stable condition $\mathcal{C}$.

THEOREM 2.3.4 (Ramakrishna). Let A be a complete commutative Noetherian local $\mathbb{Z}_{p}$-algebra and let $V_{A}$ be a finitely generated free $A$-module with an $A$ linear $G$-action. Then there exists a maximal quotient $A \rightarrow A^{\mathcal{C}}$ such that for an commutative local A-algebra $B$ of finite cardinality, the $\mathbb{Z}_{p}[G]$-module $V_{A} \otimes_{A} B$ satisfies $\mathcal{C}$ if and only if $A \rightarrow B$ factors through $A^{\mathcal{C}}$. 
Proof. This follows immediately from [Ram93, Theorem 1.1].

LEMMA 2.3.5. The inclusion functor $\mathcal{C} \rightarrow \operatorname{Mod}_{\mathbb{Z}_{p}[G]}^{\mathrm{fin}}$ has a left adjoint $(-)^{\mathcal{C}}:$ $\operatorname{Mod}_{\mathbb{Z}_{p}[G]}^{\text {fin }} \rightarrow \mathcal{C}$. For $V \in \operatorname{Mod}_{\mathbb{Z}_{p}[G]}^{\text {fin }}$, the functor $V \mapsto V^{\mathcal{C}}$ has the following additional properties:

(1) there is a quotient map $f_{V}: V \rightarrow V^{\mathcal{C}}$ of $\mathbb{Z}_{p}[G]$-modules.

(2) for $W \in \mathcal{C}$, the adjunction isomorphism

$$
\operatorname{Hom}_{\mathcal{C}}\left(V^{\mathcal{C}}, W\right) \cong \operatorname{Hom}_{\operatorname{Mod}_{Z_{p}[G]}^{\text {fin }}}(V, W)
$$

is given by $g \mapsto g \circ f_{V}$.

(3) if $A$ is a commutative $\mathbb{Z}_{p}$-algebra and $V \in \operatorname{Mod}_{A[G]}$ as well, then $V^{\mathcal{C}} \in$ $\operatorname{Mod}_{A[G]}$.

Proof. Let $V \in \operatorname{Mod}_{\mathbb{Z}_{p}[G]}^{\text {fin }}$. First we construct the quotient map $f_{V}: V \rightarrow V^{\mathcal{C}}$. Since $V$ has finite cardinality, there are a finite number of quotients of $V$. Let $\left\{V_{1}\right.$, $\left.\ldots, V_{n}\right\}$ be the (possibly empty) set all of quotients of $V$ that have property $\mathcal{C}$. Define $f_{V}: V \rightarrow V^{\mathcal{C}}$ to be the quotient by the kernel of $V \rightarrow \bigoplus_{i=1}^{n} V_{i}$. Then $V^{\mathcal{C}}$ is isomorphic to a submodule of $\bigoplus_{i=1}^{n} V_{i}$. Since $\mathcal{C}$ is closed under isomorphisms, subobjects, and finite direct sums, we see that $V^{\mathcal{C}}$ satisfies $\mathcal{C}$. By definition, any of the quotients $V \rightarrow V_{i}$ factor through $f_{V}$, and this factoring is unique since $f_{V}$ is an epimorphism.

We now let $W \in \mathcal{C}$ and show that the map given in (2) is an isomorphism. Let $W \in \mathcal{C}$ and let $f: V \rightarrow W$ be a homomorphism of $\mathbb{Z}_{p}[G]$-modules. Let $W^{\prime}=V / \operatorname{ker}(f)$, let $f^{\prime}: V \rightarrow W^{\prime}$ be the quotient map, and let $f^{\prime \prime}: W^{\prime} \rightarrow W$ be the injection induced by $f$. Since $W^{\prime}$ is isomorphic to a submodule of $W$, we have $W^{\prime} \in \mathcal{C}$. Then $f^{\prime}: V \rightarrow W^{\prime}$ must be one of the quotient maps $V \rightarrow V_{i}$, so $f^{\prime}$ factors uniquely through $f_{V}$, that is, there is a unique morphism $g^{\prime}: V^{\mathcal{C}} \rightarrow W^{\prime}$ such that $f^{\prime}=g^{\prime} \circ f_{V}$. Now let $g: V^{\mathcal{C}} \rightarrow W$ be $g=f^{\prime \prime} \circ g^{\prime}$; a simple computation shows that this assignment $f \mapsto g$ is inverse to the map given in (2).

Now we show that $V \mapsto V^{\mathcal{C}}$ is a functor. Let $h: V \rightarrow V^{\prime}$ be a $\mathbb{Z}_{p}[G]$-module homomorphism. Then $f_{V^{\prime}} \circ h \in \operatorname{Hom}_{\operatorname{Mod}_{\mathbb{Z}_{p}[G]}^{\text {in }}}\left(V,\left(V^{\prime}\right)^{\mathcal{C}}\right)$, so by (2) there is a unique map $h^{\mathcal{C}}: V^{\mathcal{C}} \rightarrow\left(V^{\prime}\right)^{\mathcal{C}}$ such that $f_{V^{\prime}} \circ h=h^{\mathcal{C}} \circ f_{V}$. This shows that $V \mapsto V^{\mathcal{C}}$ is a functor, and, together with (2), this implies that it is left adjoint to the inclusion.

Finally, (3) follows from the functoriality. Indeed, let $a \mapsto m_{a}$ denote the structure map $A \rightarrow \operatorname{End}(V)$. Then there is a unique map $m^{\mathcal{C}}: A \rightarrow \operatorname{End}\left(V^{\mathcal{C}}\right)$ such that $m_{a}^{\mathcal{C}} \circ f_{V}=f_{V} \circ m_{a}$ for all $a \in A$. The fact that $m^{\mathcal{C}}$. is a ring homomorphism follows from the fact that $f_{V}$ is an epimorphism; for example, if $a, a^{\prime} \in A$, 
we have

$$
m_{a a^{\prime}}^{\mathcal{C}} \circ f_{V}=f_{V} \circ m_{a a^{\prime}}=f_{V} \circ m_{a} \circ m_{a^{\prime}}
$$

and

$$
m_{a}^{\mathcal{C}} \circ m_{a^{\prime}}^{\mathcal{C}} \circ f_{V}=m_{a}^{\mathcal{C}} \circ f_{V} \circ m_{a^{\prime}}=f_{V} \circ m_{a} \circ m_{a^{\prime}},
$$

from which we conclude $m_{a a^{\prime}}^{\mathcal{C}}=m_{a}^{\mathcal{C}} \circ m_{a^{\prime}}^{\mathcal{C}}$.

2.4. Constructions. In what follows, we treat finite-cardinality left $E_{\bar{D}^{-}}$ modules as objects of $\operatorname{Mod}_{\mathbb{Z}_{p}[G]}^{\text {fin }}$ via the $\operatorname{map} \mathbb{Z}_{p}[G] \rightarrow E_{\bar{D}}$. By Proposition 2.1.7, $E_{\bar{D}}$ is finite as a $R_{\bar{D}}$-module. In particular, for any $i \geqslant 1$, the $\mathbb{Z}_{p}[G]$-module $E_{\bar{D}} / \mathfrak{m}_{\bar{D}}^{i} E_{\bar{D}}$ has finite cardinality, and thus is an object of $\operatorname{Mod}_{\mathbb{Z}_{p}[G]}^{\text {fin }}$.

LEMMA 2.4.1. For any $i \geqslant 1$, there is a unique $E_{\bar{D}}$-module quotient $E_{\bar{D}} / \mathfrak{m}_{\bar{D}}^{i} E_{\bar{D}} \rightarrow E_{\bar{D}}^{\mathcal{C}}(i)$ such that $E_{\bar{D}}^{\mathcal{C}}(i)$ has $\mathcal{C}$ and such that any $E_{\bar{D}^{-}}$ module quotient $E_{\bar{D}} / \mathfrak{m}_{\bar{D}}^{i} E_{\bar{D}} \rightarrow W$ where $W$ has $\mathcal{C}$ factors uniquely through $E_{\bar{D}} / \mathfrak{m}_{\bar{D}}^{i} E_{\bar{D}} \rightarrow E_{\bar{D}}^{\mathcal{C}}(i)$.

Proof. By Theorem 2.2.10, $R_{\bar{D}}[G] \rightarrow E_{\bar{D}}$ is surjective, so the lattice of $E_{\bar{D}^{-}}$ quotients and $R_{\bar{D}}[G]$-quotients of $E_{\bar{D}} / \mathfrak{m}_{\bar{D}}^{i} E_{\bar{D}}$ are identical. By Lemma 2.3.5, we can take $E_{\bar{D}}^{\mathcal{C}}(i)=\left(E_{\bar{D}} / \mathfrak{m}_{\bar{D}}^{i} E_{\bar{D}}\right)^{\mathcal{C}}$.

LEMMA 2.4.2. For any $i \geqslant 1$, there is a canonical isomorphism $E_{\bar{D}}^{\mathcal{C}}(i+1) \otimes_{R_{\bar{D}}}$ $R_{\bar{D}} / \mathfrak{m}_{\bar{D}}^{i} \stackrel{\sim}{\rightarrow} E_{\bar{D}}^{\mathcal{C}}(i)$.

Proof. Let $E^{\prime}=E_{\bar{D}}^{\mathcal{C}}(i+1) \otimes_{R_{\bar{D}}} R_{\bar{D}} / \mathfrak{m}_{\bar{D}}^{i}$. We show that $E^{\prime}$ satisfies the universal property of Lemma 2.4.1. The composite

$$
E_{\bar{D}} / \mathfrak{m}_{\bar{D}}^{i+i} E_{\bar{D}} \rightarrow E_{\bar{D}}^{\mathcal{C}}(i+1) \rightarrow E^{\prime}
$$

factors through $E_{\bar{D}} / \mathfrak{m}_{\bar{D}}^{i} E_{\bar{D}}$, so $E^{\prime}$ is a quotient of $E_{\bar{D}} / \mathfrak{m}_{\bar{D}}^{i} E_{\bar{D}}$. Since $E_{\bar{D}}^{\mathcal{C}}(i+1)$ has $\mathcal{C}$ and $E^{\prime}$ is a quotient of it, we see that $E^{\prime}$ has $\mathcal{C}$.

Now suppose that $E_{\bar{D}} / \mathfrak{m}_{\bar{D}}^{i} E_{\bar{D}} \rightarrow W$ where $W$ has $\mathcal{C}$. By the universal property of $E_{\bar{D}}^{\mathcal{C}}(i+1)$, the composite

$$
E_{\bar{D}} / \mathfrak{m}_{\bar{D}}^{i+1} E_{\bar{D}} \rightarrow E_{\bar{D}} / \mathfrak{m}_{\bar{D}}^{i} E_{\bar{D}} \rightarrow W
$$

factors uniquely through a map $E_{\bar{D}}^{\mathcal{C}}(i+1) \rightarrow W$. Since $W$ is a $R / \mathfrak{m}_{\bar{D}}^{i}$-module, this factors uniquely through $E^{\prime} \rightarrow W$.

LEMMA 2.4.3. For any $i \geqslant 1$, the module quotient $E_{\bar{D}} / \mathfrak{m}_{\bar{D}}^{i} E_{\bar{D}} \rightarrow E_{\bar{D}}^{\mathcal{C}}(i)$ has the following properties. 
(1) Let $N$ be a left $E_{\bar{D}} / \mathfrak{m}_{\bar{D}}^{i} E_{\bar{D}}$-module that has finite cardinality. Then $N$ satisfies condition $\mathcal{C}$ as a $\mathbb{Z}_{p}[G]$-module if and only if every map of left $E_{\bar{D}} / \mathfrak{m}_{\bar{D}}^{i} E_{\bar{D}}$ modules $E_{\bar{D}} / \mathfrak{m}_{\bar{D}}^{i} E_{\bar{D}} \rightarrow N$ factors through $E_{\bar{D}}^{\mathcal{C}}(i)$.

(2) There is a natural right action of $E_{\bar{D}} / \mathfrak{m}_{\bar{D}}^{i} E_{\bar{D}}$ on $E_{\bar{D}}^{\mathcal{C}}(i)$, making $E_{\bar{D}}^{\mathcal{C}}(i)$ a quotient $R_{\bar{D}}$-algebra of $E_{\bar{D}}$.

(3) An $E_{\bar{D}} / \mathfrak{m}_{\bar{D}}^{i} E_{\bar{D}}$-module $N$ that has finite cardinality satisfies condition $\mathcal{C}$ if and only if its $E_{\bar{D}}$-action factors through $E_{\bar{D}}^{\mathcal{C}}(i)$.

Proof. (1) If $N$ satisfies $\mathcal{C}$, then so does the image of any $E_{\bar{D}} / \mathfrak{m}_{\bar{D}}^{i} E_{\bar{D}} \rightarrow N$, so this arrow factors through $E_{\bar{D}}^{\mathcal{C}}(i)$ by Lemma 2.4.1. Conversely, if every such arrow factors through $E_{\bar{D}}^{\mathcal{C}}(i)$, then because of the finiteness assumption on $N$ there exists some $m \in \mathbb{Z}_{\geqslant 1}$ and a surjective map $\left(E_{\bar{D}}^{\mathcal{C}}(i)\right)^{\oplus m} \rightarrow N$. Consequently $N$ satisfies $\mathcal{C}$.

(2) Choose $z \in E_{\bar{D}}$ and consider the composite morphism of left $E_{\bar{D}}$-modules (they are also in $\operatorname{Mod}_{\mathbb{Z}_{p}[G]}^{\text {fin }}$ )

$$
E_{\bar{D}} / \mathfrak{m}_{\bar{D}}^{i} E_{\bar{D}} \stackrel{() \cdot z}{\longrightarrow} E_{\bar{D}} / \mathfrak{m}_{\bar{D}}^{i} E_{\bar{D}} \longrightarrow E_{\bar{D}}^{\mathcal{C}}(i),
$$

where the leftmost arrow is right multiplication by $z$. The composite must factor through $E_{\bar{D}}^{\mathcal{C}}(i)$ by (1). The resulting map $E_{\bar{D}}^{\mathcal{C}}(i) \rightarrow E_{\bar{D}}^{\mathcal{C}}(i)$ gives the desired right action of $z$ on $E_{\bar{D}}^{\mathcal{C}}(i)$ and shows that $E_{\bar{D}}^{\mathcal{C}}(i)$ is an $R_{\bar{D}}$-algebra.

(3) This follows directly from (1) and (2) in light of the following general fact: for an algebra $E$ and a left $E$-module $M$, the $E$-action on $M$ factors through a quotient algebra $E \rightarrow Q$ if and only if every morphism of left $E$-modules $E \rightarrow M$ factors through $Q$. This follows from the fact that any such $E \rightarrow M$ is of the form $x \mapsto x \cdot m$ for some $m \in M$.

By Lemmas 2.4.2 and 2.4.3(2), we have an inverse system $\left\{E_{\bar{D}}^{\mathcal{C}}(i)\right\}$ of $R_{\bar{D}^{-}}$ algebra quotients of $E_{\bar{D}}$. In particular, we have the $R_{\bar{D}}$-algebra quotient

$$
E_{\bar{D}} \rightarrow \lim _{i} E_{\bar{D}}^{\mathcal{C}}(i)
$$

This quotient almost gives the algebra we are looking for. However, we need to produce, from this algebra quotient, a Cayley-Hamilton algebra. The following lemma gives the general procedure for doing this. A special case of this lemma was employed in [WWE18a, WWE17a].

Lemma 2.4.5. Let $(E, D: E \rightarrow R)$ be a Cayley-Hamilton $R$-algebra. Let I $\subset$ $E$ be a two-sided ideal. Let $J \subset R$ be the ideal generated by the nonconstant 
coefficients of the polynomials in the set

$$
\left\{D_{R[t]}(1-x t) \mid x \in I\right\} \subset R[t] .
$$

Let $E^{\prime}:=E /(I, J)$ and $R^{\prime}:=R / J$, and let $f: R \rightarrow R^{\prime}$ and $g: E \rightarrow E^{\prime}$ be the quotient maps.

(1) There exists a unique Cayley-Hamilton pseudorepresentation $D^{\prime}: E^{\prime} \rightarrow R^{\prime}$ such that the pair $(f, g)$ gives a morphism $(E, D) \rightarrow\left(E^{\prime}, D^{\prime}\right)$ of CayleyHamilton algebras.

(2) For any Cayley-Hamilton representation $\rho:(E, D) \rightarrow\left(E_{A}, D_{A}: E_{A} \rightarrow A\right)$, the map $E \rightarrow E_{A}$ sends $I$ to 0 if and only if $\rho$ factors through $(E, D) \rightarrow$ $\left(E^{\prime}, D^{\prime}\right)$.

Proof. (1) The uniqueness follows from (2). To show existence, we start with the pseudorepresentation $\tilde{D}:=(f \circ D): E \otimes_{R} R^{\prime} \rightarrow R^{\prime}$. To construct $D^{\prime}$, we use Chenevier's notion of kernel of a pseudorepresentation [Che14, Section 1.17]. It is defined by the following universal property: there is an $R^{\prime}$-algebra quotient $h: E \otimes_{R} R^{\prime} \rightarrow\left(E \otimes_{R} R^{\prime}\right) / \operatorname{ker}(\tilde{D})$ and a pseudorepresentation $\tilde{D}^{\prime}:\left(E \otimes_{R}\right.$ $\left.R^{\prime}\right) / \operatorname{ker}(\tilde{D}) \rightarrow R^{\prime}$ such that $\tilde{D}=\tilde{D}^{\prime} \circ h$, and $\operatorname{ker}(\tilde{D})$ is the maximal ideal with this property.

There is an equality

$$
\operatorname{ker}(\tilde{D})=\left\{x \in E \otimes_{R} R^{\prime} \mid \tilde{D}_{R^{\prime}[t]}(1-x y t)=1 \forall y \in E \otimes_{R} R^{\prime}\right\} .
$$

This is proven in [CS16, Lemma 2.1.2]. (This lemma was removed from the final version [CS19], having become unnecessary.) Their proof uses Amitsur's formula [Ami80] for pseudorepresentations [Che14, (1.5)], together with the fact that, for any $x, y \in E, D_{R[t]}(1-x y t)=D_{R[t]}(1-y x t)$, which can be proven in the same way as for usual determinants in linear algebra.

By the definition of $J$, we have $\tilde{D}_{R^{\prime}[t]}(1-(x \otimes 1) t)=1$ for all $x \in I$, and hence $\tilde{D}_{R^{\prime}[t]}(1-(x \otimes 1) y t)=1$ for all $x \in I$ and $y \in E \otimes_{R} R^{\prime}$, since $I$ is a two-sided ideal. This implies that $I \otimes_{R} R^{\prime} \subset \operatorname{ker}(\tilde{D})$. By the property of $\operatorname{ker}(\tilde{D})$, we have $D^{\prime}: E^{\prime} \rightarrow R^{\prime}$ such that $f \circ D=D^{\prime} \circ g$.

(2) If $\rho$ factors through $(E, D) \rightarrow\left(E^{\prime}, D^{\prime}\right)$, then the map $E \rightarrow E_{A}$ factors through $E \rightarrow E^{\prime} \rightarrow E_{A}$, so $E \rightarrow E_{A}$ sends $I$ to 0 . Conversely, let $\rho:(E$, $D) \rightarrow\left(E_{A}, D_{A}\right)$ be given by $f_{A}: R \rightarrow A$ and $g_{A}: E \rightarrow E_{A}$, and assume that $g_{A}(I)=0$. To show that $\rho$ factors through $(E, D) \rightarrow\left(E^{\prime}, D^{\prime}\right)$, it suffices to show that $f_{A}(J)=0$. For any $x \in E$, the naturality of $D$ implies that the image of $D_{R[t]}(1-x t)$ in $A[t]$ under $f_{A}$ is given by $D_{A[t]}(1-x t)=\left(f_{A} \circ D\right)_{A[t]}(1-x t)$. Hence it is enough to show that, for $x \in I$, we have $\left(f_{A} \circ D\right)_{A[t]}(1-x t)=1$. 
However, since $\rho$ is a morphism of Cayley-Hamilton algebras, we have

$$
\begin{aligned}
\left(f_{A} \circ D\right)_{A[t]}(1-x t) & =\left(D_{A} \circ g_{A}\right)_{A[t]}(1-x t)=\left(D_{A}\right)_{A[t]}\left(g_{A}(1-x t)\right) \\
& =\left(D_{A}\right)_{A[t]}(1)=1,
\end{aligned}
$$

where we use the fact that $g_{A}(x)=0$.

EXAMPLE 2.4.7. Let $(E, D)$ be a Cayley-Hamilton $R$-algebra and let $I=J E$ with $J \subset R$ being an ideal. Then the Cayley-Hamilton quotient $\left(E^{\prime}, D^{\prime}\right)$ of $(E$, $D)$ by $I$ has $E^{\prime}=E / J E$ and scalar ring $R^{\prime}=R / J$. In other words, $\left(E^{\prime}, D^{\prime}\right)=$ $\left(E / J E, D \otimes_{R} R / J: E / J E \rightarrow R / J\right)$.

Definition 2.4.8. With the notation of the lemma, we call $\left(E^{\prime}, D^{\prime}\right)$ the CayleyHamilton quotient of $(E, D)$ by $I$.

DEFINITION 2.4.9. Let $K^{\mathcal{C}} \subset E_{\bar{D}}$ be the kernel of the algebra homomorphism (2.4.4). Let $\left(E_{\bar{D}}^{\mathcal{C}}, D_{E_{\bar{D}}^{\mathcal{C}}}\right)$ denote the Cayley-Hamilton quotient of $\left(E_{\bar{D}}, D_{E_{\bar{D}}}^{u}\right)$ by $K^{\mathcal{C}}$, and let $R_{\bar{D}}^{\mathcal{C}}$ denote the scalar ring of $E_{\bar{D}}^{\mathcal{C}}$.

\subsection{Extending condition $\mathcal{C}$ to pseudorepresentations and Cayley-Hamilton} representations. We extend $\mathcal{C}$ to $A[G]$-modules that may not have finite cardinality in the following way.

Definition 2.5.1. Let $\mathcal{C}$ be a stable condition on objects of $\operatorname{Mod}_{\mathbb{Z}_{p}[G]}^{\text {fin }}$. Let $\left(A, \mathfrak{m}_{A}\right)$ be a complete commutative Noetherian local $\mathbb{Z}_{p}$-algebra. For an $A[G]$ module $M$ that is finitely generated as an $A$-module, we say that $M$ satisfies condition $\mathcal{C}$ when $M / \mathfrak{m}_{A}^{i} M$ satisfies $\mathcal{C}$ for all $i \geqslant 1$.

Note that, for $\left(A, \mathfrak{m}_{A}\right)$ and $M$ as in the definition, the canonical map $M \rightarrow$ $\lim _{\leftarrow} M / \mathfrak{m}_{A}^{i} M$ is an isomorphism. Using this, one can check that this extension of

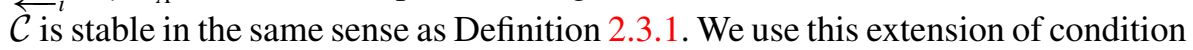
$\mathcal{C}$ without further comment.

Now we give the definition of condition $\mathcal{C}$ for Cayley-Hamilton representations.

DEFINITION 2.5.2. Let $\left(A, \mathfrak{m}_{A}\right)$ be a complete commutative Noetherian local $\mathbb{Z}_{p}$-algebra and let $(E, \rho, D)$ be a Cayley-Hamilton representation of $G$ with scalar ring $A$ and residual pseudorepresentation $\bar{D}$. We say that $(E, \rho, D)$ satisfies condition $\mathcal{C}$ if $E$ satisfies condition $\mathcal{C}$ as an $A[G]$-module. (Note that $E$ is finitely generated as an $A$-module by Proposition 2.1.7.) 
We let $\mathcal{C H}_{G, \bar{D}}^{\mathcal{C}}$ denote the full subcategory of $\mathcal{C} \mathcal{H}_{G, \bar{D}}$ whose objects satisfy condition $\mathcal{C}$.

We can be confident that this notion behaves well by finding a universal object.

THEOREM 2.5.3. The Cayley-Hamilton representation $\left(E_{\bar{D}}^{\mathcal{C}}, D_{E_{\bar{D}}^{\mathcal{C}}}: E_{\bar{D}}^{\mathcal{C}} \rightarrow R_{\bar{D}}^{\mathcal{C}}\right)$ of Definition 2.4.9 is the universal object in $\mathcal{C H}_{G, \bar{D}}^{\mathcal{C}}$.

Proof. By the definition of $\left(E_{\bar{D}}^{\mathcal{C}}, D_{E_{\bar{D}}^{\mathcal{C}}}: E_{\bar{D}}^{\mathcal{C}} \rightarrow R_{\bar{D}}^{\mathcal{C}}\right)$, we see that the map $E_{\bar{D}} \rightarrow$ $E_{\bar{D}}^{\mathcal{C}}$ sends $K^{\mathcal{C}}$ to 0 . By Lemma 2.4.3, we see that $\left(E_{\bar{D}}^{\mathcal{C}}, D_{E_{\bar{D}}^{\mathcal{C}}}: E_{\bar{D}}^{\mathcal{C}} \rightarrow R_{\bar{D}}^{\mathcal{C}}\right)$ satisfies condition $\mathcal{C}$. We now show that it has the universal property.

Let $A$ be a complete commutative Noetherian local $\mathbb{Z}_{p}$-algebra, and let $(E, \rho, D)$ be a Cayley-Hamilton representation with scalar ring $A$ and residual pseudorepresentation $\bar{D}$. We have to show that $(E, \rho, D)$ satisfies condition $\mathcal{C}$ if and only if the map of Cayley-Hamilton algebras $\left(E_{\bar{D}}, D_{E_{\bar{D}}}^{u}\right) \rightarrow(E, D)$ induced by the universal property of $\left(E_{\bar{D}}, \rho^{u}, D_{E_{\bar{D}}}^{u}\right)$ factors through $\left(E_{\bar{D}}^{\mathcal{C}}, D_{E_{\bar{D}}^{\mathcal{C}}}\right)$.

For any $i \geqslant 1, E_{\bar{D}} \rightarrow E / \mathfrak{m}_{A}^{i} E$ factors through $E_{\bar{D}} / \mathfrak{m}_{\bar{D}}^{i} E_{\bar{D}}$. (Recall that a local homomorphism of scalar rings $R_{\bar{D}} \rightarrow A$ is implicit in $\left(E_{\bar{D}}, D_{E_{\bar{D}}}^{u}\right) \rightarrow(E, D)$.)

By Lemma 2.4.3, $(E, \rho, D)$ satisfies $\mathcal{C}$ if and only if $E_{\bar{D}} / \mathfrak{m}_{\bar{D}}^{i} E_{\bar{D}} \rightarrow E / \mathfrak{m}_{A}^{i} E$ factors through $E_{\bar{D}}^{\mathcal{C}}(i)$ for every $i \geqslant 1$. Equivalently, $(E, \rho, D)$ satisfies $\mathcal{C}$ if and only if $E_{\bar{D}} \rightarrow E$ maps $K^{\mathcal{C}}$ to 0 . By Lemma 2.4.5, $K^{\mathcal{C}}$ maps to 0 in $E_{\bar{D}} \rightarrow E$ if and only if $\left(E_{\bar{D}}, D_{E_{\bar{D}}}^{u}\right) \rightarrow(E, D)$ factors through $\left(E_{\bar{D}}^{\mathcal{C}}, D_{E_{\bar{D}}^{\mathcal{C}}}\right)$.

Following the pattern of [WWE18a, Definition 5.9.1], we define condition $\mathcal{C}$ on pseudorepresentations.

DEFINITION 2.5.4. Let $A$ be an complete commutative Noetherian local $\mathbb{Z}_{p}$-algebra. Let $D: G \rightarrow A$ be a pseudorepresentation with residual pseudorepresentation $\bar{D}$. Then $D$ satisfies condition $\mathcal{C}$ provided that there exists a Cayley-Hamilton representation $\left(E, \rho, D^{\prime}\right)$ satisfying condition $\mathcal{C}$ such that $D=\psi(\rho):=D^{\prime} \circ \rho$.

We define the $\mathcal{C}$-pseudodeformation functor $\operatorname{PsDef}_{\bar{D}}^{\mathcal{C}}: \hat{\mathcal{C}}_{W(\mathbb{F})} \rightarrow$ Sets by

$$
\operatorname{PsDef}_{\bar{D}}^{\mathcal{C}}(A)=\{\text { pseudodeformations } D: G \rightarrow A \text { of } \bar{D} \text { satisfying } \mathcal{C}\} .
$$

THEOREM 2.5.5. The functor $\operatorname{PsDef}_{\bar{D}}^{\mathcal{C}}$ is represented by $R_{\bar{D}}^{\mathcal{C}}$.

Proof. Let $A \in \hat{\mathcal{C}}_{W(\mathbb{F})}$, and let $D \in \operatorname{PsDef}_{\bar{D}}(A)$. By Theorem 2.2.5, there is unique $R_{\bar{D}} \rightarrow A$ such that $D \cong D_{\bar{D}}^{u} \otimes_{R_{\bar{D}}} A$. We have to show that $D \in \operatorname{PsDef}_{\bar{D}}^{\mathcal{C}}(A)$ if and only if $R_{\bar{D}} \rightarrow A$ factors through $R_{\bar{D}} \rightarrow R_{\bar{D}}^{\mathcal{C}}$. 
If $R_{\bar{D}} \rightarrow A$ factors through $R_{\bar{D}} \rightarrow R_{\bar{D}}^{\mathcal{C}}$, then the Cayley-Hamilton representation

$$
\left(E_{\bar{D}}^{\mathcal{C}} \otimes_{R_{\bar{D}}^{\mathcal{C}}} A, \rho^{\mathcal{C}} \otimes_{R_{\bar{D}}} A: G \rightarrow\left(E_{\bar{D}}^{\mathcal{C}} \otimes_{R_{\bar{D}}^{\mathcal{C}}} A\right)^{\times}, D_{E_{\bar{D}}^{\mathcal{C}}} \otimes_{R_{\bar{D}}^{\mathcal{C}}} A\right)
$$

induces $D$ via $D=\left(R_{\bar{D}}^{\mathcal{C}} \rightarrow A\right) \circ D_{E_{\bar{D}}^{\mathcal{C}}}$ and satisfies condition $\mathcal{C}$ by Theorem 2.5.3. Consequently $D$ satisfies $\mathcal{C}$.

Now assume $D$ satisfies condition $\mathcal{C}$, that is, there exists a Cayley-Hamilton representation $\left(E, \rho, D^{\prime}\right)$ satisfying $\mathcal{C}$ such that $D=D^{\prime} \circ \rho$. By Theorem 2.5.3, there exists a morphism of Cayley-Hamilton algebras $\left(E_{\bar{D}}^{\mathcal{C}}, D_{E_{\bar{D}}^{\mathcal{C}}}\right) \rightarrow(E, D)$ inducing $\rho$. In particular, the implicit morphism of scalar rings $R_{\bar{D}}^{\mathcal{C}} \rightarrow A$ factors $R_{\bar{D}} \rightarrow A$.

2.6. Modules with Cayley-Hamilton structure. We introduce the notion of Cayley-Hamilton $G$-module.

Definition 2.6.1. Let $A \in \hat{\mathcal{C}}_{W(\mathbb{F})}$. A Cayley-Hamilton $G$-module over $A$ is the data of a Cayley-Hamilton representation $(E, \rho, D)$ of $G$ with scalar ring $A$, and an $E$-module $N$. We consider $N$ as a $A[G]$-module via the map $\rho: A[G] \rightarrow E$. We often refer to a Cayley-Hamilton $G$-module simply by the letter $N$, and call $(E, D)$ the Cayley-Hamilton algebra of $N$. We say $N$ is faithful if it is faithful as $E$-module.

EXAMPLE 2.6.2. If $N$ is an $A[G]$-module and there is a Cayley-Hamilton pseudorepresentation $D: \operatorname{End}_{A}(N) \rightarrow A$, then the canonical action of $\operatorname{End}_{A}(N)$ on $N$ makes $N$ a faithful Cayley-Hamilton $G$-module with Cayley-Hamilton algebra $\left(\operatorname{End}_{A}(N), \rho, D\right)$, where $\rho: G \rightarrow \operatorname{End}_{A}(N)$ is the action map.

EXAMPLE 2.6.3. As a special case of the previous example, suppose $N=N_{1} \oplus$ $N_{2}$ as $A$-modules such that $\operatorname{End}_{A}\left(N_{i}\right)=A$ (that is, the only endomorphisms are scalars). Note that $N_{1}$ and $N_{2}$ need not be free $A$-modules (for instance, they could be dualizing $A$-modules with $A$ non-Gorenstein; or, if $A=\mathbb{Z}_{p} \llbracket T \rrbracket$, they could be the maximal ideal). Then $\operatorname{End}_{A}(N)$ has the structure of $A$-GMA $(B, C, m)$ as in Example 3.1.7, where $B=\operatorname{Hom}_{A}\left(N_{1}, N_{2}\right)$ and $C=\operatorname{Hom}_{A}\left(N_{2}, N_{1}\right)$, and $m(f$, $g)=f \circ g$ (see Section 3, below, for a discussion of GMAs). In particular, there is a natural Cayley-Hamilton pseudorepresentation $D: \operatorname{End}_{A}(N) \rightarrow A$.

This example appears frequently in the study of pseudorepresentations associated to ordinary modular forms, where one takes $A$ to be a Hecke algebra, and $N$ to be the $p$-adic Tate module of a modular Jacobian, in which case $N$ 
is a direct sum of a free $A$-module of rank 1 and a dualizing $A$-module (see [Maz77, WWE18a, WWE17a, WWE17b]).

THEOREM 2.6.4. Let $N$ be a faithful Cayley-Hamilton $G$-module with CayleyHamilton A-algebra $(E, D)$. Then $N$ satisfies condition $\mathcal{C}$ as an $A[G]$-module if and only if $(E, \rho, D)$ satisfies condition $\mathcal{C}$ as a Cayley-Hamilton representation.

Proof. By Definition 2.5.1, it suffices to prove the theorem in the case that $A$ is Artinian and local. Choose $i \geqslant 1$ such that $\mathfrak{m}_{\bar{D}}^{i} \cdot A=0$. Let $\bar{D}: G \rightarrow \mathbb{F}$ be the residual pseudorepresentation of $D \circ \rho: G \rightarrow A$.

By Theorem 2.2.10, there is a distinguished morphism of Cayley-Hamilton algebras $\left(E_{\bar{D}}, D_{E_{\bar{D}}}^{u}\right) \rightarrow(E, D)$. Then the action map $\mathbb{Z}_{p}[G] \rightarrow \operatorname{End}_{A}(N)$ factors as

$$
\mathbb{Z}_{p}[G] \rightarrow E_{\bar{D}} \rightarrow E_{\bar{D}} / \mathfrak{m}_{\bar{D}}^{i} E_{\bar{D}} \rightarrow E \rightarrow \operatorname{End}_{A}(N) .
$$

We claim that the following are equivalent:

(1) The $\mathbb{Z}_{p}[G]$-module $N$ satisfies $\mathcal{C}$;

(2) the map $E_{\bar{D}} / \mathfrak{m}_{\bar{D}}^{i} E_{\bar{D}} \rightarrow \operatorname{End}_{A}(N)$ factors through $E_{\bar{D}}^{\mathcal{C}}(i)$;

(3) the map $E_{\bar{D}} \rightarrow \operatorname{End}_{A}(N)$ sends $K^{\mathcal{C}}$ to 0 ;

(4) the map $E_{\bar{D}} \rightarrow E$ sends $K^{\mathcal{C}}$ to 0 ;

(5) the morphism of Cayley-Hamilton algebras $\left(E_{\bar{D}}, D_{E_{\bar{D}}}^{u}\right) \rightarrow(E, D)$ factors through $\left(E_{\bar{D}}^{\mathcal{C}}, D_{E_{\bar{D}}^{\mathcal{C}}}\right)$;

(6) the Cayley-Hamilton representation $(E, D)$ satisfies condition $\mathcal{C}$.

(Recall that $K^{\mathcal{C}}$ was defined in Definition 2.4.9.) The equivalences are proven as follows:

(1) $\Longleftrightarrow(2)$ : Lemma 2.4.3(3).

$(2) \Longleftrightarrow(3)$ : From the definition of $K^{\mathcal{C}}$ and (2.6.5).

(3) $\Longleftrightarrow(4)$ : Since $N$ is faithful, the map $E \rightarrow \operatorname{End}_{A}(N)$ is injective.

(4) $\Longleftrightarrow(5)$ : Lemma 2.4.5.

$(5) \Longleftrightarrow(6)$ : Theorem 2.5.3. 
2.7. Formal moduli of representations with $\mathcal{C}$. We generalize Ramakrishna's result (Theorem 2.3.4) to any family of integral $p$-adic representations of $G$.

THEOREM 2.7.1. There exists a unique closed formal substack (respectively closed formal subscheme)

$$
\operatorname{Rep}_{G}^{\mathcal{C}, d} \subset \operatorname{Rep}_{G}^{d}, \quad\left(\text { respectively } \operatorname{Rep}_{G}^{\square, \mathcal{C}, d} \subset \operatorname{Rep}_{G}^{\square, d}\right. \text { ) }
$$

characterized by the following property. For any commutative local $\mathbb{Z}_{p}$-algebra $B$ of finite cardinality and free rank $d B$-module $V_{B}$ with a B-linear $G$-action (respectively and fixed basis), the corresponding B-point of $\operatorname{Rep}_{G}^{d}$ lies in $\operatorname{Rep}_{G}^{\mathcal{C}, d}$ (respectively of $\operatorname{Rep}_{G}^{\square, d}$ lies in $\operatorname{Rep}_{G}^{\square, \mathcal{C}, d}$ ) if and only if $V_{B}$ has $\mathcal{C}$.

Proof. It suffices to consider the case of $\operatorname{Rep}_{G}^{\square, d}$. Indeed, since condition $\mathcal{C}$ does not depend on the choice of basis, the closed subscheme $\operatorname{Rep}_{G}^{\square, \mathcal{C}, d} \subset \operatorname{Rep}_{G}^{\square, d}$ descends to a closed locus in $\operatorname{Rep}_{G}^{d}$.

By Theorem 2.2.5, we may consider a fixed residual pseudorepresentation $\bar{D}$ and produce $\operatorname{Rep}_{\bar{D}}^{\square, \mathcal{C}} \subset \operatorname{Rep}_{\bar{D}}^{\square}$. We define $\operatorname{Rep}_{\bar{D}}^{\square, \mathcal{C}}$ via the pullback diagram

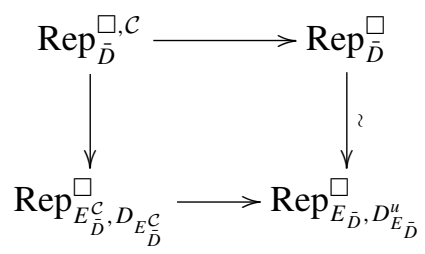

where the right vertical arrow is the isomorphism in Theorem 2.2.11.

Let $B$ be an local $\mathbb{Z}_{p}$-algebra of finite cardinality. By definition, a point $V_{B} \in \operatorname{Rep}_{\bar{D}}^{\square}(B)$ lies in $\operatorname{Rep}_{\bar{D}}^{\square, \mathcal{C}}(B)$ if and only if the map $\left(E_{\bar{D}}, D_{E_{\bar{D}}}^{u}\right) \rightarrow$ $\left(\operatorname{End}_{B}\left(V_{B}\right)\right.$, det) factors through $\left(E_{\bar{D}}^{\mathcal{C}}, D_{E_{\bar{D}}^{\mathcal{C}}}\right)$. By Theorem 2.5.3, this occurs if and only if the Cayley-Hamilton representation $\left(\operatorname{End}_{B}\left(V_{B}\right)\right.$, det) has $\mathcal{C}$, which, by Theorem 2.6.4, is if and only if $V_{B}$ has $\mathcal{C}$. By Lemma 2.2.1, this characterizes $\operatorname{Rep}_{\bar{D}}^{\square, \mathcal{C}}$.

Corollary 2.7.2. Let $A \in \operatorname{Tfg}_{\mathbb{Z}_{p}}$ and $\left(V_{A}, \rho_{A}\right) \in \operatorname{Rep}_{G}^{d}(A)$. There exists a unique quotient morphism $A \rightarrow A^{\mathcal{C}}$ in $\mathrm{Tfg}_{\mathbb{Z}_{p}}$ such that, for any local A-algebra $B$ of finite cardinality, the object $V_{A} \otimes_{A} B$ of $\operatorname{Mod}_{\mathbb{Z}_{p}[G]}^{\text {fin }}$ satisfies $\mathcal{C}$ if and only if the homomorphism $A \rightarrow B$ factors through $A^{\mathcal{C}}$. 
Proof. The quotient $A \rightarrow A^{\mathcal{C}}$ is defined by the pullback square

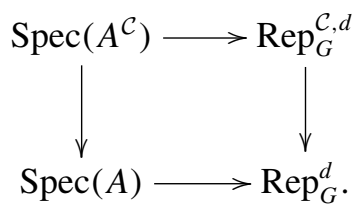

The characterizing property follows from the characterizing property of $\operatorname{Rep}_{G}^{\mathcal{C}, d}$.

\section{Deformation conditions for generalized matrix algebras}

For the remainder of the paper, we assume that the pseudorepresentation $\bar{D}$ is multiplicity-free (see Definition 3.2.1). Under this assumption, as was first noticed by Chenevier [Che14], the universal Cayley-Hamilton algebra with residual pseudorepresentation $\bar{D}$ admits the additional structure of a generalized matrix algebra (GMA). Generalized matrix algebras, which were first introduced by Bellaïche and Chenevier [BC09], are a particularly concrete and explicit type of Cayley-Hamilton algebra.

In this section, we recall in Section 3.1 the theory of generalized matrix algebras and in Section 3.2 why the universal Cayley-Hamilton algebra admits this structure. In Section 3.3, we exploit this extra structure to prove that the universal Cayley-Hamilton algebra with property $\mathcal{C}$, constructed in Section 2, is characterized by representations. This is the main new result of the section. Explicitly, in the multiplicity-free case, we show that, if every representation with $\mathcal{C}$ factors through a quotient of universal Cayley-Hamilton algebra, then that quotient must be universal Cayley-Hamilton algebra with property $\mathcal{C}$.

The results of Sections 3.1 and 3.2 will also be used in Section 4, where we exploit the GMA structure to compute the Cayley-Hamilton algebra with property $\mathcal{C}$ in terms of group cohomology.

3.1. Generalized matrix algebras and their adapted representations. A generalized matrix algebra is a particular kind of Cayley-Hamilton algebra with extra data. We learned this notion from Bellaïche-Chenevier [BC09].

Definition 3.1.1 [BC09, Section 1.3]. Let $R$ be a ring. A generalized matrix algebra over $R$ (or an $R-G M A$ ) is the data of:

(1) an $R$-algebra $E$ that is finitely generated as an $R$-module;

(2) a set of orthogonal idempotents $e_{1}, \ldots, e_{r} \in E$ such that $\sum_{i} e_{i}=1$; and 
(3) a set of isomorphisms of $R$-algebras $\phi_{i}: e_{i} E e_{i} \stackrel{\sim}{\rightarrow} M_{d_{i}}(R)$ for $i=1, \ldots, r$.

We call $\mathcal{E}=\left(\left\{e_{i}\right\},\left\{\phi_{i}\right\}\right)$ the data of idempotents or GMA structure of $E$, and write the $R$-GMA as $(E, \mathcal{E})$. We call the list of integers $\left(d_{1}, \ldots, d_{r}\right)$ the type of $(E, \mathcal{E})$. These data are required to satisfy the condition that the function $\operatorname{Tr}_{\mathcal{E}}: E \rightarrow A$ defined by

$$
\operatorname{Tr}_{\mathcal{E}}(x):=\sum_{i=1}^{r} \operatorname{tr}\left(\phi_{i}\left(e_{i} x e_{i}\right)\right)
$$

is a central function, that is, $\operatorname{Tr}_{\mathcal{E}}(x y)=\operatorname{Tr}_{\mathcal{E}}(y x)$ for all $x, y \in E$.

Given an $R$-GMA $(E, \mathcal{E})$ and an $R^{\prime}$-GMA $\left(E^{\prime}, \mathcal{E}^{\prime}\right)$, a morphism of GMAs $\rho$ : $(E, \mathcal{E}) \rightarrow\left(E^{\prime}, \mathcal{E}^{\prime}\right)$ is the data of a ring homomorphism $f: R \rightarrow R^{\prime}$ and morphism of $R$-algebras $\rho: E \rightarrow E^{\prime}$ such that $\mathcal{E}$ and $\mathcal{E}^{\prime}$ are of the same type $\left(d_{1}, \ldots, d_{r}\right)$, we have $g\left(e_{i}\right)=e_{i}^{\prime}$ and $f \circ \phi_{i}=\phi_{i}^{\prime} \circ g$ for $i=1, \ldots, r$.

EXAMPLE 3.1.2. The matrix algebra $M_{d}(R)$ comes with a natural $R$-GMA structure $\mathcal{E}=\left(1\right.$, id : $\left.M_{d}(R) \stackrel{\sim}{\rightarrow} M_{d}(R)\right)$ of type $(d)$. More generally, given any ordered partition of $d=d_{1}+\cdots+d_{r}$ of $d$, there is a natural $R$-GMA structure $\mathcal{E}_{\text {block }}$ on $M_{d}(R)$ of type $\left(d_{1}, \ldots, d_{r}\right)$. Namely, the natural $R$-algebra map with block-diagonal image

$$
v_{1} \times \cdots \times v_{r}: M_{d_{1}}(R) \times \cdots \times M_{d_{r}}(R) \hookrightarrow M_{d}(R)
$$

induces $\mathcal{E}_{\text {block }}=\left(e_{i}=v_{i}\left(1_{i}\right), \phi_{i}\right)$, where $1_{i} \in M_{d_{i}}(R)$ is the identity matrix, and $\phi_{i}$ is the inverse to $v_{i}$ on its image $e_{i} M_{d}(R) e_{i}$.

LEMMA 3.1.3. Given an $R-G M A(E, \mathcal{E})$, there is a canonical Cayley-Hamilton pseudorepresentation $D_{\mathcal{E}}: E \rightarrow R$, such that $\operatorname{Tr}_{D_{\mathcal{E}}}=\operatorname{Tr}_{\mathcal{E}}$. A morphism of $R$ GMAs $(E, \mathcal{E}) \rightarrow\left(E^{\prime}, \mathcal{E}^{\prime}\right)$ induces a morphism of Cayley-Hamilton algebras $(E$, $\left.D_{\mathcal{E}}\right) \rightarrow\left(E^{\prime}, D_{\mathcal{E}^{\prime}}\right)$.

Proof. See [WE18a, Proposition 2.23]; its statement includes the first claim. The second claim follows from examining the formula for $D_{\mathcal{E}}$ given in [WE18a], noting that a morphism of GMAs preserves the idempotents that are used to specify $D_{\mathcal{E}}$.

This lemma gives a faithful embedding of the category of $R$-GMAs into the category of $R$-Cayley-Hamilton algebras. We consider this embedding as an inclusion. We extend the definition of Cayley-Hamilton representation to GMAs as follows. 
DEFINITION 3.1.4. If $\left(E^{\prime}, \mathcal{E}^{\prime}\right)$ is a GMA, we refer to a morphism of pseudorepresentations $(E, D) \rightarrow\left(E^{\prime}, D_{\mathcal{E}^{\prime}}\right)$ as a $G M A$ representation of $(E, D)$. If $(E, \mathcal{E})$ is another GMA, we call a GMA representation $\left(E, D_{\mathcal{E}}\right) \rightarrow\left(E^{\prime}, D_{\mathcal{E}^{\prime}}\right)$ adapted if the same data give a morphism of GMAs $(E, \mathcal{E}) \rightarrow\left(E^{\prime}, \mathcal{E}^{\prime}\right)$.

LEMMA 3.1.5. Given an R-GMA $(E, \mathcal{E})$ of type $\left(d_{1}, \ldots, d_{r}\right)$, we can associate to it the data of

(1) $R$-modules $\mathcal{A}_{i, j}$ for $1 \leqslant i, j \leqslant r$,

(2) canonical isomorphisms $\mathcal{A}_{i, i} \stackrel{\sim}{\rightarrow} R$ for $1 \leqslant i \leqslant r$, and

(3) $R$-module homomorphisms $\varphi_{i, j, k}: \mathcal{A}_{i, j} \otimes_{R} \mathcal{A}_{j, k} \rightarrow \mathcal{A}_{i, k}$ for $1 \leqslant i, j, k \leqslant r$,

such that $\left(\mathcal{A}_{i, j}, \varphi_{i, j, k}\right)$ completely determine $(E, \mathcal{E})$ and there is an isomorphism of $R$-modules

$$
E \stackrel{\sim}{\longrightarrow}\left(\begin{array}{cccc}
M_{d_{1}}\left(\mathcal{A}_{1,1}\right) & M_{d_{1} \times d_{2}}\left(\mathcal{A}_{1,2}\right) & \cdots & M_{d_{1} \times d_{r}}\left(\mathcal{A}_{1, r}\right) \\
M_{d_{2} \times d_{1}}\left(\mathcal{A}_{2,1}\right) & M_{d_{2}}\left(\mathcal{A}_{2,2}\right) & \cdots & M_{d_{2} \times d_{r}}\left(\mathcal{A}_{2, r}\right) \\
\vdots & \vdots & \vdots & \vdots \\
M_{d_{r} \times d_{1}}\left(\mathcal{A}_{r, 1}\right) & M_{d_{2}}\left(\mathcal{A}_{r, 2}\right) & \cdots & M_{d_{r}}\left(\mathcal{A}_{r, r}\right)
\end{array}\right),
$$

Moreover, the collection of maps $\varphi_{i, j, k}$ satisfies properties (UNIT), (COM), and (ASSO) given in [BC09, Section 1.3.2], and there is a bijection between R-GMAs of type $\left(d_{1}, \ldots, d_{r}\right)$ and data $\left(\mathcal{A}_{i, j}, \varphi_{i, j, k}\right)$ satisfying (UNIT), (COM), and (ASSO).

Proof. This is explained in [BC09, Sections 1.3.1-1.3.6]. The association is given as follows. Let $E_{i}:=\phi_{i}^{-1}\left(\delta^{1,1}\right)$, where $\delta^{1,1}$ denotes the elementary matrix with 1 as $(1,1)$ th entry, and 0 otherwise. Define $\mathcal{A}_{i, j}:=E_{j} E E_{i}$. The maps $\varphi_{i, j, k}$ are induced by the multiplication in $E$. In particular, note that $\phi_{i}$ induces a canonical isomorphism $\mathcal{A}_{i, i} \stackrel{\sim}{\rightarrow} R$.

We will not spell out the bijection or the properties (UNIT), (COM), and (ASSO) in general. Instead, we explain the content of the lemma in the case of type $(1,1)$.

EXAMPLE 3.1.7. There is a bijection between $R$-GMAs $(E, \mathcal{E})$ of type $(1,1)$ and triples $(B, C, m)$ where $B, C$ are finitely generated $R$-modules and $m: B \otimes_{R} C \rightarrow$ $R$ is an $R$-module homomorphism, such that the squares 

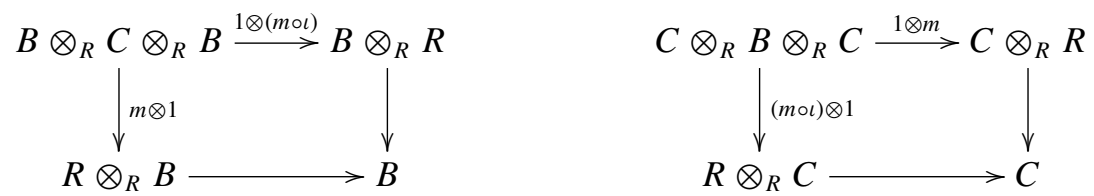

commute. Here $\iota: C \otimes_{R} B \stackrel{\sim}{\rightarrow} B \otimes_{R} C$ is the isomorphism given by $b \otimes c \mapsto c \otimes b$, and the unlabeled maps are the $R$-action maps.

The $R$-GMA associated to a triple $(B, C, m)$ is

$$
E=\left(\begin{array}{ll}
R & B \\
C & R
\end{array}\right)
$$

This means that $E=R \oplus B \oplus C \oplus R$ as an $R$-module, and the multiplication on $E$ is given by $2 \times 2$-matrix multiplication, but where the action maps $R \otimes_{R} B \rightarrow B$, $R \otimes_{R} C \rightarrow C$ and the map $m$ are used in place of the scalar multiplication. The idempotents $e_{1}, e_{2}$ are given in this notation by $e_{1}=\left(\begin{array}{ll}1 & 0 \\ 0 & 0\end{array}\right)$ and $e_{2}=\left(\begin{array}{ll}0 & 0 \\ 0 & 1\end{array}\right)$, and the isomorphisms $\phi_{i}$ are given by the identifications $\left(\begin{array}{ll}R & 0 \\ 0 & 0\end{array}\right) \stackrel{\sim}{\rightarrow} R$ and $\left(\begin{array}{ll}0 & 0 \\ 0 & R\end{array}\right) \stackrel{\sim}{\rightarrow} R$.

In the notation of the lemma, $\mathcal{A}_{1,2}=B$ and $\mathcal{A}_{2,1}=C$, and the maps $\varphi_{i, j, k}$ are the action maps, except $\varphi_{1,2,1}=m$ and $\varphi_{2,1,2}=m \circ \iota$. Indeed, the (UNIT) property requires that the maps $\varphi_{i, j, k}$ equal the action maps, unless $(i, j, k) \in\{(1,2,1),(2$, $1,2)\}$. The (ASSO) property is expressed by the commutative squares above, and the $(\mathrm{COM})$ property is that $\varphi_{2,1,2}=\varphi_{1,2,1} \circ \iota$.

EXAMPLE 3.1.9. In the foregoing example, setting $m$ to be the zero map is always a valid choice.

Definition 3.1.10 [BC09, Definition 1.3.6]. Let $A$ be a commutative $R$-algebra and let $(E, \mathcal{E})$ be an $R$-GMA of type $\left(d_{1}, \ldots, d_{r}\right)$. Let $d=\sum_{i=1}^{r} d_{i}$ and let $\left(M_{d}(A), \mathcal{E}_{\text {block }}\right)$ be the $A$-GMA of type $\left(d_{1}, \ldots, d_{r}\right)$ constructed in Example 3.1.2.

An adapted representation of $(E, \mathcal{E})$, denoted $\rho:(E, \mathcal{E}) \rightarrow M_{d}(A)$ is a morphism of GMAs $\rho:(E, \mathcal{E}) \rightarrow\left(M_{d}(A), \mathcal{E}_{\text {block }}\right)$. A pseudorepresentation $D: E \otimes_{R} A \rightarrow A$ is adapted to $\mathcal{E}$ if $D=D_{\mathcal{E}} \otimes_{R} A$. When the data of idempotents $\mathcal{E}$ is understood, we say $D$ is adapted instead of adapted to $\mathcal{E}$.

Now fix $(E, \mathcal{E})=\left(E,\left(\left\{e_{i}\right\},\left\{\phi_{i}\right\}\right)\right)$, an $R$-GMA of type $\left(d_{1}, \ldots, d_{r}\right)$, and let $\left(\mathcal{A}_{i, j}, \varphi_{i, j, k}\right)$ be the data associated to it by Lemma 3.1.5. We define a set-valued functor on commutative $R$-algebras by

$$
\operatorname{Rep}_{(E, \mathcal{E}), \mathrm{GMA}}^{\square}: A \mapsto\left\{\text { Adapted representations } \rho:(E, \mathcal{E}) \rightarrow M_{d}(A)\right\}
$$


There is an explicit presentation for $\operatorname{Rep}_{(E, \mathcal{E}), \mathrm{GMA}}^{\square}$ as an affine Spec $R$-scheme as follows.

THEOREM 3.1.11. The functor $\operatorname{Rep}_{(E, \mathcal{E}), \mathrm{GMA}}^{\square}$ is represented by Spec $S$, where $S$ is an R-algebra quotient

$$
\operatorname{Sym}_{R}^{*}\left(\bigoplus_{1 \leqslant i \neq j \leqslant r} \mathcal{A}_{i, j}\right) \rightarrow S
$$

by the ideal generated by the set of all $\varphi(b \otimes c)-b \otimes c$, with $b \in \mathcal{A}_{i, j}, c \in \mathcal{A}_{j, k}$ and $\varphi=\varphi_{i, j, k}$ for all $1 \leqslant i, j, k \leqslant r$. In particular, $S$ is a finitely generated $R$-algebra. Moreover, for $1 \leqslant i, j \leqslant r$, the natural $R$-module maps $\mathcal{A}_{i, j} \rightarrow S$ are split injections of $R$-modules, inclusive of the case $R \stackrel{\sim}{\rightarrow} \mathcal{A}_{i, i} \hookrightarrow S$, which is the $R$ algebra structure map of $S$. The universal adapted representation $\rho_{\mathrm{GMA}}:(E$, $\mathcal{E}) \rightarrow M_{d}(S)$ is given by the isomorphism of (3.1.6) along with these R-module injections. In particular, the R-algebra homomorphism $E \rightarrow M_{d}(S)$ is injective.

Proof. See [BC09, Proposition 1.3.9] and its proof, as well as [BC09, Proposition 1.3.13] for the split injectivity.

By [WE18a, Proposition 2.23], any adapted representation of $(E, \mathcal{E})$ is compatible with $D_{\mathcal{E}}$. This determines a monomorphism $\operatorname{Rep}_{(E, \mathcal{E}), \mathrm{GMA}}^{\square} \hookrightarrow \operatorname{Rep}_{E, D_{\mathcal{E}}}^{\square}$, which can easily be observed to be a closed immersion.

Let $\rho^{\square}$ denote the universal object of $\operatorname{Rep}_{E, D_{\mathcal{E}}}^{\square}$. Let $Z(\mathcal{E}) \subset \mathrm{GL}_{d}$ denote the subgroup that stabilizes $\rho^{\square}\left(e_{i} E e_{i}\right)$, for all $i=1, \ldots, r$, under the adjoint action of $\mathrm{GL}_{d}$ on $\operatorname{Rep}_{E, D_{\mathcal{E}}}^{\square}$. This $Z(\mathcal{E})$ is a torus of rank $r$.

Proposition 3.1.12 [WE18a, Theorem 2.27]. For any $R-G M A(E, \mathcal{E})$, the map $\operatorname{Rep}_{(E, \mathcal{E}), \mathrm{GMA}}^{\square} \hookrightarrow \operatorname{Rep}_{E, D_{\mathcal{E}}}^{\square}$ induces an isomorphism

$$
\left[\operatorname{Rep}_{(E, \mathcal{E}), \mathrm{GMA}}^{\square} / Z(\mathcal{E})\right] \stackrel{\sim}{\longrightarrow} \operatorname{Rep}_{E, D_{\mathcal{E}}}
$$

of Spec $R$-algebraic stacks.

3.2. Residually multiplicity-free representations of profinite groups. Let $G$ be a profinite group satisfying the $\Phi_{p}$ finiteness condition, and let $\mathbb{F}$ be a finite field of characteristic $p$ with algebraic closure $\overline{\mathbb{F}}$.

By [WE18a, Corollary 2.9(2)], which is a mild strengthening of [Che14, Theorem 2.16], for any pseudorepresentation $\bar{D}: G \rightarrow \mathbb{F}$, there is a unique semisimple representation $\rho_{\bar{D}}^{s s}: G \rightarrow \mathrm{GL}_{d}(\mathbb{F})$ such that $\bar{D}=\operatorname{det} \circ \rho_{\bar{D}}^{s s}$. 
Definition 3.2.1. A residual pseudorepresentation $\bar{D}: G \rightarrow \mathbb{F}$ is multiplicityfree if $\rho_{\bar{D}}^{s s} \otimes_{\mathbb{F}} \overline{\mathbb{F}}$ has pairwise nonisomorphic simple factors, and each of these factors is defined over $\mathbb{F}$. Equivalently, $\rho_{\bar{D}}^{s s}$ is a direct sum of pairwise nonisomorphic absolutely irreducible representations. A representation $\left(V_{A}\right.$, $A) \in \operatorname{Rep}_{G}^{d}(A)$ is residually multiplicity-free if $\left(V_{A}, A\right) \in \operatorname{Rep}_{\bar{D}}(A)$ with $\bar{D}$ multiplicity-free.

Note that when $\rho_{\bar{D}}^{s s} \otimes_{\mathbb{F}} \overline{\mathbb{F}}$ has distinct simple factors, then $\rho_{\bar{D}}^{s s}$ is multiplicity-free after replacing $\mathbb{F}$ with a finite extension.

THEOREM 3.2.2. Let $\bar{D}: G \rightarrow \mathbb{F}$ be multiplicity-free, and let $\left(d_{1}, \ldots, d_{r}\right)$ be the dimensions of the simple factors of $\rho_{\bar{D}}^{s s}$. Let $A$ be a Noetherian Henselian local ring with residue field $\mathbb{F}$, and let $(E, \rho, D)$ Cayley-Hamilton representation over $A$ with residual pseudorepresentation $\bar{D}$. Then there is an A-GMA structure $\mathcal{E}$ of type $\left(d_{1}, \ldots, d_{r}\right)$ on $E$ such that $D=D_{\mathcal{E}}$.

Moreover, given a morphism $(E, \rho, D) \rightarrow\left(E^{\prime}, \rho^{\prime}, D^{\prime}\right)$ of such objects, the structures $\mathcal{E}$ and $\mathcal{E}^{\prime}$ may be chosen so that the map $(E, \mathcal{E}) \rightarrow\left(E^{\prime}, \mathcal{E}^{\prime}\right)$ is a morphism of GMAs.

Proof. The structure $\mathcal{E}$ is constructed in [Che14, Theorem 2.22(ii)], and it follows from this construction that $D=D_{\mathcal{E}}$ (see [WE18a, Theorem 2.27]). Moreover, the construction only depends on the choice certain lifts of idempotents. If we first choose the structure $\mathcal{E}=\left(\left\{e_{i}\right\},\left\{\phi_{i}\right\}\right)$ on $E$, then the images of $e_{i}$ in $E^{\prime}$ will give a choice of lifts of idempotents in $E^{\prime}$, and for the resulting GMA structure $\mathcal{E}^{\prime}$, the map $(E, \mathcal{E}) \rightarrow\left(E^{\prime}, \mathcal{E}^{\prime}\right)$ is a morphism of GMAs.

For the rest of this section, we fix a pseudorepresentation $\bar{D}: G \rightarrow \mathbb{F}$ that is multiplicity-free. By Theorem 2.2.5, the ring $R_{\bar{D}}$ is Noetherian and complete (and hence Henselian). By Theorem 3.2.2, we can and do fix a choice of $A$-GMA structure $\mathcal{E}_{\bar{D}}$ of type $\left(d_{1}, \ldots, d_{r}\right)$ on $E_{\bar{D}}$ such that $D_{E_{\bar{D}}}^{u}=D_{\mathcal{E}_{\bar{D}}}$.

COROllary 3.2.3. Assume that $\bar{D}$ is multiplicity-free, and let $\mathcal{E}_{\bar{D}}$ be choice of $R_{\bar{D}}$-GMA structure on $E_{\bar{D}}$ as in Theorem 3.2.2.

(1) There are isomorphisms

$$
\left[\operatorname{Rep}_{\left(E_{\bar{D}}, \mathcal{E}_{\bar{D}}\right), \mathrm{GMA}}^{\square} / Z\left(\mathcal{E}_{\bar{D}}\right)\right] \stackrel{\sim}{\longrightarrow} \operatorname{Rep}_{E_{\bar{D}}, D_{\bar{E}_{\bar{D}}}^{u}} \stackrel{\sim}{\longrightarrow} \operatorname{Rep}_{\bar{D}}
$$

of stacks on $\mathrm{Tfg}_{\mathbb{Z}_{p}}$. 
(2) Let $B$ be a commutative Noetherian local $\mathbb{Z}_{p}$-algebra. Given an adapted representation $\left(E_{\bar{D}}, \mathcal{E}_{\bar{D}}\right) \rightarrow M_{d}(B)$, the map $E_{\bar{D}} \rightarrow M_{d}(B)$ is a compatible representation of $\left(E_{\bar{D}}, D_{E_{\bar{D}}}^{u}\right)$.

(3) Let $(E, \rho, D)$ be a Cayley-Hamilton representation of $G$ with residual pseudorepresentation $\bar{D}$. Then there is an $A-G M A$ structure $\mathcal{E}$ on $E_{A}$ such that $D_{\mathcal{E}}=D$ and such that the map $\left(E_{\bar{D}}, D_{\mathcal{E}_{\bar{D}}}\right) \rightarrow\left(E, D_{\mathcal{E}}\right)$ is adapted.

REMARK 3.2.4. Let $(E, \mathcal{E})$ be an $R$-GMA where $R \in \operatorname{Tfg}_{\mathbb{Z}_{p}}$ is local. Let $S$ be the $R$-algebra from Theorem 3.1.11 so that $\operatorname{Rep}_{(E, \mathcal{E}), \mathrm{GMA}}^{\square}=\operatorname{Spec}(S)$. Restricting the functor $\operatorname{Rep}_{(E, \mathcal{E}), \mathrm{GMA}}^{\square}$ to the subcategory $\operatorname{Tfg}_{R}$ of the category of $R$-algebras, we obtain an affine formal scheme $\operatorname{Spf}(\hat{S})$, where $\hat{S}$ is the $\mathfrak{m}_{R} S$-adic completion of $S$. We also denote $\operatorname{Spf}(\hat{S})$ by $\operatorname{Rep}_{(E, \mathcal{E}), \mathrm{GMA}}^{\square}$, abusing notation. This is how we consider $\left[\operatorname{Rep}_{\left(E_{\bar{D}}, \mathcal{E}_{\bar{D}}\right), \mathrm{GMA}}^{\square} / Z\left(\mathcal{E}_{\bar{D}}\right)\right]$ as a formal stack on $\operatorname{Tfg}_{\mathbb{Z}_{p}}$.

Proof. Statement (1) follows from Proposition 3.1.12 and Theorem 2.2.11, while (2) follows from the statement of Theorem 3.2.2 that $D_{E_{\bar{D}}}^{u}=D_{\mathcal{E}_{\bar{D}}}$. Statement (3) follows from the second part of Theorem 3.2.2.

Lemma 3.2.5. Assume that $\bar{D}$ is multiplicity-free. Let $\left(E_{\bar{D}}, D_{E_{\bar{D}}}^{u}\right) \rightarrow(E, D)$ be a morphism of Cayley-Hamilton algebras, where $E$ is an R-algebra.

(1) For any nonzero $x \in E$, there is a commutative local $R$-algebra $B$ of finite cardinality and a compatible representation $\rho_{B}: E \rightarrow M_{d}(B)$ such that $\rho_{B}(x) \neq 0$.

(2) Let $\left(E_{\bar{D}}, D_{E_{\bar{D}}}^{u}\right) \rightarrow\left(E^{\prime}, D^{\prime}\right)$ be another morphism of Cayley-Hamilton algebras, and assume that $E_{\bar{D}} \rightarrow E$ and $E_{\bar{D}} \rightarrow E^{\prime}$ are surjective. If, for all $B$ as in (1) and all compatible representations $\rho_{B}: E_{\bar{D}} \rightarrow M_{d}(B)$, the map $\rho_{B}$ factors through $E$ if and only if it factors through $E^{\prime}$, then there is a canonical isomorphism $(E, D) \stackrel{\sim}{\rightarrow}\left(E^{\prime}, D^{\prime}\right)$ of Cayley-Hamilton algebras.

Proof. Since $\bar{D}$ is multiplicity-free, we may fix a GMA structure $\mathcal{E}_{\bar{D}}$ on $E_{\bar{D}}$ as in Theorem 3.2.2. By Corollary 3.2.3(3), this gives a GMA structure $\mathcal{E}$ on $E$ such that $\left(E_{\bar{D}}, D_{E_{\bar{D}}}^{u}\right) \rightarrow(E, D)$ induces a morphisms of GMAs $\left(E_{\bar{D}}, \mathcal{E}_{\bar{D}}\right) \rightarrow(E$, $\mathcal{E})$, and similarly for $\left(E^{\prime}, D^{\prime}\right)$. By Corollary 3.2.3(3) we may work with adapted representations of these GMAs in the place of compatible representations of the Cayley-Hamilton algebras. We do this for the remainder of the proof.

(1) By Theorem 3.1.11 and Remark 3.2.4, there is $\hat{S} \in \operatorname{Tfg}_{R}$ such that $\operatorname{Rep}_{(E, \mathcal{E}), \mathrm{GMA}}^{\square}=\operatorname{Spf}(\hat{S})$ and, moreover, $E \hookrightarrow M_{d}(S)$ splits as an $R$-module map. Therefore, $\rho_{\mathrm{GMA}}: E \rightarrow M_{d}(\hat{S})$ remains injective. 
Let $x \in E$ be a nonzero element, so $\rho_{\mathrm{GMA}}(x) \neq 0$. Let $y \in \hat{S}$ be a nonzero entry in the matrix $\rho_{\mathrm{GMA}}(x)$. By Lemma 2.2.1, there is a commutative local $R$ algebra $B$ of finite cardinality and an $R$-algebra homomorphism $f: \hat{S} \rightarrow B$ such that $f(y) \neq 0$. Then the composite $f \circ \rho_{\mathrm{GMA}}: E \rightarrow M_{d}(B)$ is an adapted representation such that $\left(f \circ \rho_{\mathrm{GMA}}\right)(x) \neq 0$.

(2) Since the maps $\left(E_{\bar{D}}, D_{E_{\bar{D}}}^{u}\right) \rightarrow(E, D)$ and $\left(E_{\bar{D}}, D_{E_{\bar{D}}}^{u}\right) \rightarrow\left(E^{\prime}, D^{\prime}\right)$ are morphisms of pseudorepresentations and the maps $g: E_{\bar{D}} \rightarrow E$ and $g^{\prime}: E_{\bar{D}} \rightarrow E^{\prime}$ are surjective, it suffices to show $\operatorname{ker}(g)=\operatorname{ker}\left(g^{\prime}\right)$. Assume for a contradiction that there exists $x \in \operatorname{ker}(g)$ with $x \notin \operatorname{ker}\left(g^{\prime}\right)$. Since $g^{\prime}(x) \neq 0$, part (1) implies that there is a commutative local $R$-algebra $B$ of finite cardinality and a compatible representation $\rho_{B}^{\prime}: E^{\prime} \rightarrow M_{d}(B)$ such that $\rho_{B}^{\prime}\left(g^{\prime}(x)\right) \neq 0$. The adapted representation $\tilde{\rho}_{B}=\rho_{B}^{\prime} \circ g^{\prime}: E_{\bar{D}} \rightarrow M_{d}(B)$ factors through $E^{\prime}$, so it must factor through $E$ by assumption. This implies that $\tilde{\rho}_{B}=\rho_{B} \circ g$ for some adapted representation $\rho_{B}$ of $E$. But then

$$
0=\rho_{B}(g(x))=\tilde{\rho}_{B}(x)=\rho_{B}^{\prime}\left(g^{\prime}(x)\right) \neq 0
$$

a contradiction.

3.3. Condition $\mathcal{C}$ in the residually multiplicity-free case. Let $G$ be a profinite group satisfying the $\Phi_{p}$ finiteness condition. Fix a stable condition $\mathcal{C} \subset$ $\operatorname{Mod}_{\mathbb{Z}_{p}[G]}^{\text {in }}$ as in Definition 2.3.1 and a residual pseudorepresentation $\bar{D}: G \rightarrow \mathbb{F}$. By Theorem 2.5.3, there is a universal Cayley-Hamilton algebra with condition $\mathcal{C}$, denoted $\left(E_{\bar{D}}^{\mathcal{C}}, D_{E_{\bar{D}}^{\mathcal{C}}}\right)$. In the case that $\bar{D}$ is multiplicity-free, the following theorem gives an alternate characterization of $\left(E_{\bar{D}}^{\mathcal{C}}, D_{E_{\bar{D}}^{\mathcal{C}}}\right)$.

THEOREM 3.3.1. Assume that $\bar{D}$ is multiplicity-free.

(1) Let $B$ be a commutative local $R_{\bar{D}}$-algebra of finite cardinality. Let $\rho_{B}:\left(E_{\bar{D}}\right.$, $\left.D_{E_{\bar{D}}}^{u}\right) \rightarrow M_{d}(B)$ be a compatible representation, and let $V_{B} \cong B^{d}$ denote the corresponding object of $\operatorname{Mod}_{\mathbb{Z}_{p}[G]}^{\text {in }}$. Then $V_{B}$ satisfies $\mathcal{C}$ if and only if $\rho_{B}$ factors through $E_{\bar{D}}^{\mathcal{C}}$.

(2) The property of (1) characterizes the quotient $E_{\bar{D}} \rightarrow E_{\bar{D}}^{\mathcal{C}}$.

Proof. Part (1) follows from Theorems 2.6.4 and 2.5.3. Part (2) is immediate from Lemma 3.2.5(2).

When $\bar{D}$ is not multiplicity-free, we only know how to characterize $E_{\bar{D}}^{\mathcal{C}}$ by Theorem 2.5.3; compare [BC09, Section 1.3.4]. 
This theorem furnishes a convenient way to prove that, in certain cases where a pseudorepresentation comes from a representation, property $\mathcal{C}$ for the pseudorepresentation is related to property $\mathcal{C}$ for a representation inducing it.

COROLLARY 3.3.2. Let $\bar{D}$ be multiplicity-free with $\bar{\rho}_{\bar{D}}^{s s}=\bar{\rho}_{1} \oplus \cdots \bar{\rho}_{r}$ over $\mathbb{F}$, where the $\bar{\rho}_{i}$ are absolutely irreducible and pairwise nonisomorphic of dimension $d_{i}$. For $i=1, \ldots, r$, let $\rho_{i}: G \rightarrow M_{d_{i}}(A)$ be a deformation of $\bar{\rho}_{i}$, and let $D=$ $\psi\left(\rho_{1} \oplus \cdots \oplus \rho_{r}\right)$. Then $D$ has $\mathcal{C}$ if and only if there are deformations $\rho_{i}^{\prime}$ of $\bar{\rho}_{i}$ with $\mathcal{C}$ for $i=1, \ldots, r$ such that $D=\psi\left(\rho_{1}^{\prime} \oplus \cdots \oplus \rho_{r}^{\prime}\right)$.

Proof. If $D=\psi\left(\rho_{1}^{\prime} \oplus \cdots \oplus \rho_{r}^{\prime}\right)$ for such $\rho_{i}^{\prime}$, then its clear that $D$ has $\mathcal{C}$. Now assume $D$ has $\mathcal{C}$, so, by definition, there is a Cayley-Hamilton representation ( $E$, $\left.\rho, D^{\prime}\right)$ of $G$ that has $\mathcal{C}$ such that $D=D^{\prime} \circ \rho$. Since $\bar{D}$ is multiplicity-free, we may assume that $D^{\prime}=D_{\mathcal{E}}$ for some GMA structure $\mathcal{E}=\left(\left\{e_{i}\right\},\left\{\phi_{i}\right\}\right)$ on $E$ such that $\rho_{i}^{\prime}=e_{i} \rho e_{i}: G \rightarrow M_{d_{i}}(A)$ is a deformation of $\bar{\rho}_{i}$. Replacing $E$ by the image of $A[G] \rightarrow E$ if necessary, we may assume that the maps $\rho_{i, j}$ given by the GMA structure are surjective.

Now, the fact that $D=\psi\left(\rho_{1} \oplus \cdots \oplus \rho_{r}\right)$ implies that $\phi_{i, j, k}=0$ for all triples $i, j, k$ of distinct integers with $1 \leqslant i, j, k \leqslant r$ (see [BC09, Section 1.5.1]). Then the sum of projection maps

$$
E \rightarrow \bigoplus_{i=1}^{r} e_{i} E e_{i}=\bigoplus_{i=1}^{r} M_{d_{i}}(A)
$$

is an $A$-algebra homomorphism. The resulting map

$$
G \rightarrow E \rightarrow M_{d}(A)
$$

is $\rho^{\prime}:=\bigoplus_{i=1}^{r} \rho_{i}^{\prime}$. Since $\left(E, D^{\prime}\right)$ has $\mathcal{C}$, the map $E_{\bar{D}} \rightarrow E$ factors through $E_{\bar{D}}^{\mathcal{C}}$. This implies that $\rho^{\prime}$ has $\mathcal{C}$ by Theorem 3.3.1(1), and hence each $\rho_{i}^{\prime}$ has $\mathcal{C}$ by stability.

REMARK 3.3.3. The preceding corollary can be useful in proving automorphy lifting theorems. Indeed, it is a general version of an argument used in the proof of $\left[\mathrm{ACC}^{+}\right.$18, Proposition 4.4.6].

WARNING 3.3.4. When applying these results to the situation of Section 5.3 below (deformations of global Galois representations, where $\mathcal{C}$ is a condition on the local representation), the preceding corollary only applies to deformations $D$ that are globally reducible. For example, take $\mathcal{C}$ to be the condition 'de Rham at $p^{\prime}$ and $\bar{D}=\psi\left(\mathbb{F}_{p}(1) \oplus \mathbb{F}_{p}\right): G_{\mathbb{Q}} \rightarrow \mathbb{F}_{p}$. Suppose $D=\psi(\rho)$ where $\rho: G_{\mathbb{Q}} \rightarrow \mathrm{GL}_{2}\left(\mathbb{Z}_{p}\right)$ is irreducible, but $\left.\rho\right|_{G_{p}}$ is a nonsplit extension of $\mathbb{Z}_{p}(1)$ by $\mathbb{Z}_{p}$ 
(and hence not de Rham). Then $\left.D\right|_{G_{p}}=\psi\left(\left.\rho\right|_{G_{p}}\right)=\psi\left(\mathbb{Z}_{p}(1) \oplus \mathbb{Z}_{p}\right)$ is a sum of two de Rham characters, and hence de Rham, but $D$ is not (globally) de Rham.

If $\bar{\rho}$ is absolutely irreducible, let $R_{\bar{\rho}}^{\mathcal{C}}$ be the deformation-with- $\mathcal{C}$ ring defined by Ramakrishna (denoted $R_{\mathcal{C}}(\bar{\rho})$ in [Ram93, Proposition 1.2]) and let $\rho_{\bar{\rho}}^{\mathcal{C}}$ be the universal deformation.

COROLlary 3.3.5. Suppose that $\bar{\rho}_{\bar{D}}^{s s}=\bar{\rho}$ is absolutely irreducible. Then there is canonical isomorphism $R_{\bar{D}}^{\mathcal{C}} \rightarrow R_{\bar{\rho}}^{\mathcal{C}}$ determined by the pseudodeformation $\psi\left(\rho_{\bar{\rho}}^{\mathcal{C}}\right)$ of $\bar{D}$.

Proof. It is well known that $R_{\bar{D}}=R_{\bar{\rho}}$-that is, every deformation $D$ of $\bar{D}$ is of the form $D=\psi(\rho)$ for a unique deformation $\rho$ of $\bar{\rho}$. (Using pseudocharacters, this is due to Carayol and Mazur. For pseudorepresentations, this is proved by Chenevier [Che14, Theorem 2.22(i)]; it also follows from Theorem 3.2.2.) In this situation, by the previous corollary, $D$ has $\mathcal{C}$ if and only if $\rho$ has $\mathcal{C}$, so the two quotients $R_{\bar{D}}^{\mathcal{C}}$ and $R_{\bar{\rho}}^{\mathcal{C}}$ of $R_{\bar{D}}=R_{\bar{\rho}}$ coincide.

This corollary shows that our $R_{\bar{D}}^{\mathcal{C}}$ is really a generalization of Ramakrishna's theory.

\section{Deformation conditions and extensions}

In [BC09], Bellaïche and Chenevier used the explicit nature of the generalized matrix algebras discussed in the previous section to partially compute the universal pseudodeformation ring in terms of extension groups, in the category $\operatorname{Mod}_{\mathbb{Z}_{p}[G]}$, of the constituent irreducible representations of the residual representation. This calculation is a generalization of the description of the tangent space of the deformation ring of an irreducible representation in terms of adjoint cohomology.

In this section, we show that pseudodeformations-with- $\mathcal{C}$ may be controlled by similar calculations, but where the extension groups are taken in the category $\mathcal{C}$. Whereas Bellaïche and Chenevier work with arbitrary multiplicity-free $\bar{D}$, we, for reasons of simplicity and clarity, have chosen only to consider the case where $\bar{D}$ is a sum of two characters (but see Remark 4.3.6).

In this situation, where $\bar{D}=\psi\left(\bar{\chi}_{1} \oplus \bar{\chi}_{2}\right)$ for distinct characters $\bar{\chi}_{1}$ and $\bar{\chi}_{2}$, we now outline what we can compute about $R_{\bar{D}}^{\mathcal{C}}$. First, we consider a simpler deformation ring $R_{\bar{D}}^{\text {red, } \mathcal{C}}$ where we only consider deformations $D$ that remain reducible (that is, they have the form $\left.D=\psi\left(\chi_{1} \oplus \chi_{2}\right)\right)$. This ring can be computed in terms of deformation rings of the characters $\bar{\chi}_{1}$ and $\bar{\chi}_{2}$. Next, we wish to 
compute the kernel $J^{\mathcal{C}}=\operatorname{ker}\left(R_{\bar{D}}^{\mathcal{C}} \rightarrow R_{\bar{D}}^{\text {red, } \mathcal{C}}\right)$, which is called the reducibility ideal. By the work of Bellaïche and Chenevier, this ideal can be described as a quotient of $B^{\mathcal{C}} \otimes_{R_{\bar{D}}^{\mathcal{C}}} C^{\mathcal{C}}$, where these modules come from the GMA structure on $E_{\bar{D}}^{\mathcal{C}}$. Finally, $B^{\mathcal{C}} / J^{\mathcal{C}} B^{\mathcal{C}}$ and $C^{\mathcal{C}} / J^{\mathcal{C}} C^{\mathcal{C}}$ can be described in terms of extension groups in the category $\mathcal{C}$ of the universal deformations $\chi_{1}$ and $\chi_{2}$. In total, this gives an upper bound for the size of $J^{\mathcal{C}} /\left(J^{\mathcal{C}}\right)^{2}$ in terms of extension groups of $\chi_{1}$ and $\chi_{2}$ in the category $\mathcal{C}$, where $R_{\bar{D}}^{\mathcal{C}} / J^{\mathcal{C}}$ is a quotient of $R_{\bar{D}}^{\mathcal{C}}$ that is fairly simple.

REMARK 4.0.1. When proving modularity-lifting theorems, this is often the kind of upper bound one needs. In that situation, one has a surjection $R_{\bar{D}}^{\mathcal{C}} \rightarrow \mathbb{T}$, where $\mathbb{T}$ is the quotient of 'modular deformations'. To prove modularity, one needs to show that $R_{\bar{D}}^{\mathcal{C}}$ is not 'too big', giving an upper bound on the size of $R_{\bar{D}}^{\mathcal{C}}$. In this case, the extension groups in $\mathcal{C}$ are given as certain Selmer groups in Galois cohomology (see Example 4.3.3). Often, the size of these Selmer groups can be bounded in terms of modular invariants (for example, zeta values), and the resulting upper bound on $J^{\mathcal{C}} /\left(J^{\mathcal{C}}\right)^{2}$ can be enough to show that $R_{\bar{D}}^{\mathcal{C}} \rightarrow \mathbb{T}$ is injective. This is the strategy of [WWE17b, WWE18b].

REMARK 4.0.2. In fact, our results allow a similar computation about $I / I^{2}$ for any ideal $I$ containing $J^{\mathcal{C}}$. In particular, taking $I$ to be the maximal ideal of $R_{\bar{D}}^{\mathcal{C}}$, we get a description of the (co)tangent space of $R_{\bar{D}}^{\mathcal{C}}$. This computation about $J^{\mathcal{C}} /\left(J^{\mathcal{C}}\right)^{2}$ is giving information about the 'relative tangent space' over the reducible deformation ring, and is, in practice, often more useful.

We now outline the contents of this section. In Section 4.2, we review the results of Bellaïche-Chenevier. In Section 4.3, we prove our generalization to deformations-with- $\mathcal{C}$.

4.1. Conventions. Throughout this section, we fix $G$, a profinite group satisfying the $\Phi_{p}$ finiteness condition, and two distinct characters $\bar{\chi}_{1}$, $\bar{\chi}_{2}: G \rightarrow \mathbb{F}^{\times}$. We let $\bar{D}=\psi\left(\bar{\chi}_{1} \oplus \bar{\chi}_{2}\right)$.

By Theorem 3.2.2, we can and do fix a $R_{\bar{D}}$-GMA structure $\mathcal{E}_{\bar{D}}=\left(\left\{e_{1}, e_{2}\right\},\left\{\phi_{1}\right.\right.$, $\left.\left.\phi_{2}\right\}\right)$ on $E_{\bar{D}}$. We write $\left(E_{\bar{D}}, \mathcal{E}_{\bar{D}}\right)$ and $\rho^{u}: G \rightarrow E_{\bar{D}}^{\times}$as

$$
E_{\bar{D}} \cong\left(\begin{array}{ll}
R_{\bar{D}} & B^{u} \\
C^{u} & R_{\bar{D}}
\end{array}\right), \quad \rho^{u}(\sigma)=\left(\begin{array}{cc}
\rho_{1,1}^{u}(\sigma) & \rho_{1,2}^{u}(\sigma) \\
\rho_{2,1}^{u}(\sigma) & \rho_{2,2}^{u}(\sigma)
\end{array}\right)
$$

and write $m: B^{u} \otimes_{R_{\bar{D}}} C^{u} \rightarrow R_{\bar{D}}$ for the induced map, as in Example 3.1.7. For $b \in B^{u}$ and $c \in C^{u}$, we define $b \cdot c=m(b \otimes c) \in R_{\bar{D}}$. We can and do assume that the idempotents are ordered so that the image of $\rho_{i, i}(\sigma)$ under $R_{\bar{D}} \rightarrow \mathbb{F}$ is $\bar{\chi}_{i}(\sigma)$. 
By Corollary 3.2.3(3), a Cayley-Hamilton representation $(E, \rho, D)$ of $G$ with residual pseudorepresentation $\bar{D}$ inherits a GMA structure from the data above. We use matrix notation for $E$ and $\rho$ according to this structure, as in (4.1.1) (for example, writing the coordinates of $\rho$ as $\rho_{i, j}$ ).

4.2. Review of reducibility, extensions, and GMAs. In this subsection, we review known results of [BC09, Section 1.5] relating the structure of GMAs to Ext-groups.

Definition 4.2.1. Let $A \in \hat{\mathcal{C}}_{W(\mathbb{F})}$. We call a pseudodeformation $D: G \rightarrow A$ of $\bar{D}$ reducible if $D=\psi\left(\chi_{1} \oplus \chi_{2}\right)$ for characters $\chi_{i}: G \rightarrow A^{\times}$deforming $\bar{\chi}_{i}$. Otherwise, we call $D$ irreducible.

A GMA representation $\left(E, \rho: G \rightarrow E^{\times}, D_{\mathcal{E}}\right)$ of $G$ with residual pseudorepresentation $\bar{D}$ is called reducible (respectively irreducible) provided that pseudodeformation $D_{\mathcal{E}} \circ \rho: G \rightarrow A$ is reducible (respectively irreducible).

Proposition 4.2.2. Let $A$ be a commutative Noetherian local $\mathbb{Z}_{p}$-algebra. Let $D: G \rightarrow$ A pseudorepresentation with residual pseudorepresentation $\bar{D}$.

(1) $D$ is reducible if and only if $D=\psi(\rho)$ for some GMA representation $\rho$ with scalar ring $A$ such that $\rho_{1,2}(G) \cdot \rho_{2,1}(G)$ is zero.

(2) Let $J=B^{u} \cdot C^{u} \subset R_{\bar{D}}$ be the image ideal of $B^{u} \otimes_{R_{\bar{D}}} C^{u}$ in $R_{\bar{D}}$ under $m$ : $B^{u} \otimes_{R_{\bar{D}}} C^{u} \rightarrow R_{\bar{D}}$. Then $D$ is reducible if and only if the corresponding local homomorphism $R_{\bar{D}} \rightarrow$ A kills $J$.

Proof. See [BC09, Section 1.5.1].

In light of Proposition 4.2.2, we establish the following terminology.

Definition 4.2.3. The ideal $J \subset R_{\bar{D}}$ of Proposition 4.2.2(2) is called the reducibility ideal of $R_{\bar{D}}$. The image of $J$ under the map $R_{\bar{D}} \rightarrow A$ corresponding to a pseudodeformation $D: G \rightarrow A$ of $\bar{D}$ is called the reducibility ideal of $D$.

We define $E_{\bar{D}}^{\text {red }}$ to be the Cayley-Hamilton quotient of $E_{\bar{D}}$ by $J E_{\bar{D}}$, which as in Example 2.4.7 is the usual algebra quotient $E_{\bar{D}}^{\text {red }}=E_{\bar{D}} / J E_{\bar{D}}$. Its scalar ring is $R_{\bar{D}}^{\text {red }}=R_{\bar{D}} / J$ and is called the universal reducible pseudodeformation ring for $\bar{D}$. We let $\left(E_{\bar{D}}^{\text {red }}, \rho^{\text {red }}, \mathcal{E}_{\bar{D}}^{\text {red }}\right)$ denote the corresponding GMA representation of $G$, and write the decomposition arising from (4.1.1) as

$$
E_{\bar{D}}^{\mathrm{red}}=\left(\begin{array}{ll}
R_{\bar{D}}^{\mathrm{red}} & B^{\mathrm{red}} \\
C^{\mathrm{red}} & R_{\bar{D}}^{\mathrm{red}}
\end{array}\right) .
$$


WARNING 4.2.5. Being reducible implies that the map $m: B^{\text {red }} \otimes C^{\text {red }} \rightarrow R_{\bar{D}}^{\text {red }}$ is zero, but it does not imply that either $B^{\text {red }}$ or $C^{\text {red }}$ is zero. In fact, for a fixed $g \in G$, it is possible that both $\rho_{1,2}^{\text {red }}(g) \in B^{\text {red }}$ and $\rho_{2,1}^{\text {red }}(g) \in C^{\text {red }}$ are nonzero.

Let for $i=1,2$, let $R_{\bar{\chi}_{i}}$ denote the universal deformation ring of $\bar{\chi}_{i}$ and let $\chi_{i}^{u}: G \rightarrow R_{\bar{\chi}_{i}}^{\times}$denote the universal deformation.

\section{PROPOSITION 4.2.6.}

(1) If $\rho: G \rightarrow(E, D)$ is a GMA representation of $G$ with scalar ring $A$ and residual pseudorepresentation $\bar{D}$, then the resulting GMA map $\left(E_{\bar{D}}, \mathcal{E}_{\bar{D}}\right) \rightarrow$ $(E, \mathcal{E})$ factors through $\left(E_{\bar{D}}^{\mathrm{red}}, \mathcal{E}_{\bar{D}}^{\mathrm{red}}\right)$ if and only if $\rho$ is reducible.

(2) There is a canonical isomorphism $R_{\bar{D}}^{\mathrm{red}} \cong R_{\bar{\chi}_{1}} \hat{\otimes}_{W(\mathbb{F})} R_{\bar{\chi}_{2}}$. Letting $\chi_{i}=\chi_{i}^{u} \otimes_{R_{\bar{\chi}_{i}}}$ $R_{\bar{D}}^{\text {red }}$, the universal reducible pseudodeformation of $\bar{D}$ is $\psi\left(\chi_{1} \oplus \chi_{2}\right)$.

(3) In terms of the decomposition (4.2.4), we have $\rho_{i, i}^{\mathrm{red}}=\chi_{i}$.

Proof. (1) By Lemma 2.4.5, the GMA map $\left(E_{\bar{D}}, \mathcal{E}_{\bar{D}}\right) \rightarrow(E, \mathcal{E})$ factors through $\left(E_{\bar{D}}^{\text {red }}, \mathcal{E}_{\bar{D}}^{\text {red }}\right)$ if and only if $E_{\bar{D}} \rightarrow E$ sends $J E_{\bar{D}}$ to zero. Because $R \rightarrow E$ is injective whenever there is a pseudorepresentation $D: E \rightarrow R$ [WWE18a, Lemma 5.2.5], $J E_{\bar{D}}$ maps to zero in $E$ if and only if $R_{\bar{D}} \rightarrow A$ factors through $R_{\bar{D}}^{\text {red }}$, which, by Proposition 4.2.2(2), is equivalent to $D \circ \rho$ being reducible.

(2) By Yoneda's lemma, it suffices to construct a canonical functorial isomorphism

$$
\operatorname{Hom}\left(R_{\bar{D}}^{\text {red }}, A\right) \cong \operatorname{Hom}\left(R_{\bar{\chi}_{1}} \hat{\otimes}_{W(\mathbb{F})} R_{\bar{\chi}_{2}}, A\right)
$$

for $A \in \hat{\mathcal{C}}_{W(\mathbb{F})}$. Given $R_{\bar{\chi}_{1}} \hat{\otimes}_{W(\mathbb{F})} R_{\bar{\chi}_{2}} \rightarrow A$, we define a reducible deformation $D$ of $\bar{D}$ by $D=\psi\left(\chi_{1}^{u} \otimes A \oplus \chi_{2}^{u} \otimes A\right)$, which determines an element of $\operatorname{Hom}\left(R_{\bar{D}}^{\text {red }}, A\right)$ by Proposition 4.2.2(2) and the universal property of $R_{\bar{D}}$.

Conversely, given $R_{\bar{D}}^{\text {red }} \rightarrow A$, consider the GMA representation $\rho^{\text {red }} \otimes_{R_{\bar{D}}^{\text {red }}} A$. Since $B^{\text {red }} \cdot C^{\text {red }}=0$, we have that $\rho_{i, i}^{\text {red }} \otimes_{R_{\bar{D}}^{\text {red }}} A: G \rightarrow A^{\times}($for $i=1,2)$ is a character that, by the conventions of Section 4.1, is a deformation of $\bar{\chi}_{i}$. This pair of characters determines an element of $\operatorname{Hom}\left(R_{\bar{\chi}_{1}} \hat{\otimes}_{W(\mathbb{F})} R_{\bar{\chi}_{2}}, A\right)$.

We have defined maps between $\operatorname{Hom}\left(R_{\bar{D}}^{\text {red }}, A\right)$ and $\operatorname{Hom}\left(R_{\bar{\chi}_{1}} \hat{\otimes}_{W(\mathbb{F})} R_{\bar{\chi}_{2}}, A\right)$. The reader can check these are mutually inverse and functorial in $A$.

(3) It follows from the construction of the isomorphism in (2).

The following key result relates $R_{\bar{D}}^{\text {red }}[G]$-module extensions to the structure of $E_{\bar{D}}^{\text {red }}$. For ease of notation, we write $\chi_{i}$ for the base change from $R_{\overline{\chi_{i}}}$ to $R_{\bar{D}}^{\text {red }}$ of the universal deformation $\chi_{i}^{u}$ of $\bar{\chi}_{i}$. 
Proposition 4.2.7. Let $A \in \hat{\mathcal{C}}_{W(\mathbb{F})}$ and let $M$ be a finitely generated A-module. For $i=1,2$, let $\chi_{i, A}: G \rightarrow A^{\times}$be characters deforming $\bar{\chi}_{i}$. By Proposition 4.2.6, this induces a unique local homomorphism $R_{\bar{D}}^{\mathrm{red}} \rightarrow A$. There is a natural isomorphism

$$
\operatorname{Hom}_{A}\left(B^{\text {red }} \otimes_{R_{\bar{D}}^{\text {red }}} A, M\right) \stackrel{\sim}{\longrightarrow} \operatorname{Ext}_{A[G]}^{1}\left(\chi_{2, A}, \chi_{1, A} \otimes_{A} M\right)
$$

as well as a similar isomorphism in the C-coordinate.

Proof. The details may be found in [WWE17a, Lemma 4.1.5, proof of (4.1.7)], see also [BC09, Theorem 1.5.6]. We reproduce the construction of the map here with notation that will be convenient in Section 4.3.

Let $E_{M}=\left(\begin{array}{cc}A & M \\ 0 & A\end{array}\right)$, with GMA structure as in Example 3.1.9. Given a homomorphism $f: B^{\text {red }} \otimes_{R_{\bar{D}}^{\text {red }}} A \rightarrow M$, we have morphism of GMAs $E_{\bar{D}}^{\text {red }} \otimes_{R_{\bar{D}}^{\text {red }}} A \rightarrow E_{M}$ as the composition

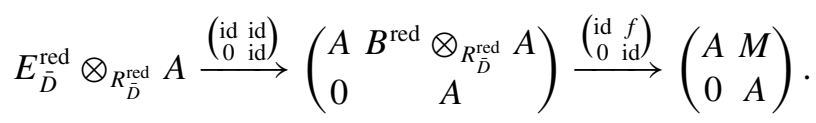

Using the fact that $B^{\text {red }} \cdot C^{\text {red }}=0$, we see that $e_{1} E_{M} e_{2}=\left(\begin{array}{cc}0 & M \\ 0 & 0\end{array}\right)$ is a left $E_{\bar{D}}$-submodule of $E_{M} e_{2}=\left(\begin{array}{ll}0 & M \\ 0 & A\end{array}\right)$. Noting that $e_{1} E_{M} e_{2} \cong \chi_{1, A} \otimes_{A} M$ and $E_{M} e_{2} / e_{1} E_{M} e_{2} \cong \chi_{2, A}$ as $A[G]$-modules, we obtain an exact sequence

$$
0 \longrightarrow \chi_{1, A} \otimes_{A} M \rightarrow E_{M} e_{2} \longrightarrow \chi_{2, A} \longrightarrow 0,
$$

which determines the corresponding element of $\operatorname{Ext}_{A[G]}^{1}\left(\chi_{2, A}, \chi_{1, A} \otimes_{A} M\right)$. Conversely, any such extension can be realized in the form $E_{M} e_{2}$.

\subsection{GMA structures corresponding to extensions with an abstract property.} Let $\mathcal{C} \subset \operatorname{Mod}_{\mathbb{Z}_{p}[G]}^{\text {in }}$ be a stable property, as in Definition 2.3.1. The following lemma is a well-known consequence of stability, and we leave the proof to the reader. To state the lemma clearly, we introduce some notation for extension classes. If $E$ is an algebra and $V_{1}, V_{2}$ are $E$-modules, given an extension class $c \in \operatorname{Ext}_{E}^{1}\left(V_{1}, V_{2}\right)$, and an exact sequence

$$
0 \rightarrow V_{2} \rightarrow V \rightarrow V_{1} \rightarrow 0
$$

representing $c$, we call $V$ an extension module for $c$.

Let $V_{1}, V_{2} \in \mathcal{C}$, and assume that $V_{1}, V_{2} \in \operatorname{Mod}_{R[G]}$ as well for commutative $\mathbb{Z}_{p^{-}}$ algebra $R$. Define $\operatorname{Ext}_{R[G], \mathcal{C}}^{1}\left(V_{1}, V_{2}\right)$ as the subset of $\operatorname{Ext}_{R[G]}^{1}\left(V_{1}, V_{2}\right)$ consisting of extension classes $c$ such that, for every extension module $V$ for $c$, we have $V \in \mathcal{C}$. 
LEMMA 4.3.2. With $V_{1}, V_{2}$ as above, we have the following.

(1) For a class $c \in \operatorname{Ext}_{R[G]}^{1}\left(V_{1}, V_{2}\right)$, if some extension module $V$ for $c$ has $V \in \mathcal{C}$, then $c \in \operatorname{Ext}_{R[G], \mathcal{C}}^{1}\left(V_{1}, V_{2}\right)$.

(2) The subset $\operatorname{Ext}_{R[G], \mathcal{C}}^{1}\left(V_{1}, V_{2}\right) \subset \operatorname{Ext}_{R[G]}^{1}\left(V_{1}, V_{2}\right)$ is a sub-R-module.

EXAMPLE 4.3.3. Let $H_{1}, \ldots, H_{n} \subset G$ be subgroups, and, for $i=1, \ldots, n$, let $\mathcal{C}_{i} \subset \operatorname{Mod}_{\mathbb{Z}_{p}\left[H_{i}\right]}^{\text {fin }}$ be a stable condition. Assume that the condition $\mathcal{C} \subset \operatorname{Mod}_{\mathbb{Z}_{p}[G]}^{\text {fin }}$ arises from the $\mathcal{C}_{i}$ as in Example 2.3.3. Then $\operatorname{Ext}_{R[G], \mathcal{C}}^{1}\left(V_{1}, V_{2}\right)$ is the kernel of the map

$$
\operatorname{Ext}_{R[G]}^{1}\left(V_{1}, V_{2}\right) \rightarrow \bigoplus_{i=1}^{n} \frac{\operatorname{Ext}_{R\left[H_{i}\right]}^{1}\left(V_{1}, V_{2}\right)}{\operatorname{Ext}_{R\left[H_{i}\right], \mathcal{C}_{i}}^{1}\left(V_{1}, V_{2}\right)}
$$

given by the restrictions $\operatorname{Ext}_{R[G]}^{1}\left(V_{1}, V_{2}\right) \rightarrow \operatorname{Ext}_{R\left[H_{i}\right]}^{1}\left(V_{1}, V_{2}\right)$ followed by the quotients. (This is sometimes referred to as a Selmer group.)

Let $E_{\bar{D}}^{\mathcal{C}}$ be as in Theorem 3.3.1, and let $E_{\bar{D}}^{\mathcal{C} \text {,red }}=E_{\bar{D}}^{\mathcal{C}} / J E_{\bar{D}}^{\mathcal{C}}$ where $J \subset R_{\bar{D}}$ is the reducibility ideal. Following the notation of (4.1.1), we write them as

$$
E_{\bar{D}}^{\mathcal{C}}=\left(\begin{array}{ll}
R_{\bar{D}}^{\mathcal{C}} & B^{\mathcal{C}} \\
C^{\mathcal{C}} & R_{\bar{D}}^{\mathcal{C}}
\end{array}\right), \quad E_{\bar{D}}^{\mathcal{C} \text {,red }}=\left(\begin{array}{ll}
R_{\bar{D}}^{\mathcal{C}, \text { red }} & B^{\mathcal{C}, \text { red }} \\
C^{\mathcal{C}, \text { red }} & R_{\bar{D}}^{\mathcal{C}, \text { red }}
\end{array}\right)
$$

We denote the Cayley-Hamilton representations by $\rho^{\mathcal{C}}: G \rightarrow\left(E_{\bar{D}}^{\mathcal{C}}\right)^{\times}$and $\rho^{\mathcal{C} \text {,red }}$ : $G \rightarrow\left(E_{\bar{D}}^{\mathcal{C}, \text { red }}\right)^{\times}$.

By Ramakrishna's result [Ram93, Proposition 1.2], for $i=1,2$, there is a quotient $R_{\bar{\chi}_{i}} \rightarrow R_{\bar{\chi}_{i}}^{\mathcal{C}}$ that represents the functor of deformations of $\bar{\chi}_{i}$ having property $\mathcal{C}$ and $\chi_{i}^{u, \mathcal{C}}=\chi_{i}^{u} \otimes_{R_{\bar{x}_{i}}} R_{\bar{\chi}_{i}}^{\mathcal{C}}$ is the universal deformation with property $\mathcal{C}$.

Proposition 4.3.4. There is a canonical commutative diagram

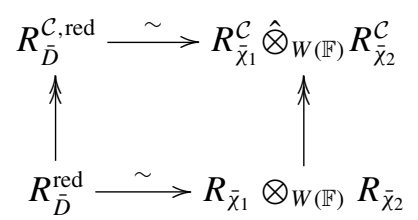

where the vertical maps are the quotients and the lower horizontal map is the isomorphism of Proposition 4.2.6(2). 
Proof. For simplicity of notation, let $R=R_{\bar{\chi}_{1}}^{\mathcal{C}} \hat{\otimes}_{W(\mathbb{F})} R_{\bar{\chi}_{2}}^{\mathcal{C}}$ and, for $i=1,2$, let $\chi_{i}^{\mathcal{C}}=\chi_{i}^{u, \mathcal{C}} \otimes_{R_{\bar{\chi}_{i}}^{\mathcal{C}}} R$.

By Proposition 4.2.6(2), the composite map $R_{\bar{D}}^{\text {red }} \rightarrow R$ corresponds to the reducible pseudodeformation $D=\psi\left(\chi_{1}^{\mathcal{C}} \oplus \chi_{2}^{\mathcal{C}}\right)$. The $G$-module $N=\chi_{1}^{\mathcal{C}} \oplus \chi_{2}^{\mathcal{C}}$ is a faithful Cayley-Hamilton $G$-module with Cayley-Hamilton algebra $\left(E=\left(\begin{array}{ll}R & 0 \\ 0 & R\end{array}\right)\right.$, $\left.D_{\mathcal{E}}\right)$. By Theorem 2.6.4, the map $\left(E_{\bar{D}}, D_{E_{\bar{D}}}^{u}\right) \rightarrow\left(E, D_{\mathcal{E}}\right)$ factors through $E_{\bar{D}}^{\mathcal{C}}$. Since $D$ is reducible, Proposition 4.2.2 implies that it further factors through $E_{\bar{D}}^{\mathcal{C}, \text { red }}$. This implies that $R_{\bar{D}}^{\text {red }} \rightarrow R$ factors through $R_{\bar{D}}^{\mathcal{C}, \text { red }}$.

On the other hand, the composite map $R_{\bar{\chi}_{1}} \otimes_{W(\mathbb{F})} R_{\bar{\chi}_{2}} \rightarrow R_{\bar{D}}^{\mathcal{C} \text {,red }}$ corresponds to the pair of characters $e_{1} \rho^{\text {red, } \mathcal{C}} e_{1}, e_{2} \rho^{\text {red, } \mathcal{C}} e_{2}: G \rightarrow R_{\bar{D}}^{\mathcal{C} \text {,red }}$. Since these characters are quotient $R_{\bar{D}}^{\text {red }}[G]$-modules of the $R_{\bar{D}}^{\text {red }}[G]$-module $E_{\bar{D}}^{\mathcal{C} \text {,red }}$, which has $\mathcal{C}$, we see that they both have $\mathcal{C}$ as well. This implies that the map $R_{\bar{\chi}_{1}} \otimes_{W(\mathbb{F})} R_{\bar{\chi}_{2}} \rightarrow R_{\bar{D}}^{\mathcal{C} \text {,red }}$ factors through $R$, completing the proof.

We write $\chi_{i}^{\mathcal{C}}$ for the base change from $R_{\bar{\chi}_{i}}^{\mathcal{C}}$ to $R_{\bar{D}}^{\mathcal{C} \text {,red }}$ of the universal deformation of $\bar{\chi}_{i}$ satisfying $\mathcal{C}$.

THEOREM 4.3.5. Let $A \in \hat{\mathcal{C}}_{W(\mathbb{F})}$ and let $M$ be a finitely generated $A$-module. For $i=1,2$, let $\chi_{i, A}: G \rightarrow A^{\times}$be characters deforming $\bar{\chi}_{i}$ and having property $\mathcal{C}$. By Proposition 4.3.4, this induces a unique local homomorphism $R_{\bar{D}}^{\text {red,C }} \rightarrow A$. There is a natural isomorphism

$$
\operatorname{Hom}_{A}\left(B^{\mathcal{C}, \text { red }} \otimes_{R_{\bar{D}}^{\text {red, } \mathcal{C}}} A, M\right) \stackrel{\sim}{\longrightarrow} \operatorname{Ext}_{A[G], \mathcal{C}}^{1}\left(\chi_{2, A}, \chi_{1, A} \otimes_{A} M\right),
$$

as well as a similar isomorphism in the C-coordinate.

REMARK 4.3.6. Throughout this section, we have restricted our attention to twodimensional pseudorepresentations and GMAs of type $(1,1)$. However, in [BC09, Theorem 1.5.6], Bellaïche and Chenevier prove a version of Proposition 4.2.7 for GMAs of any dimension $d$ and any type $\left(d_{1}, \ldots, d_{r}\right)$.

We have made this choice strictly for clarity of exposition. We fully expect a version of Theorem 4.3.5 to be true for GMAs of any dimension $d$ and any type $\left(d_{1}, \ldots, d_{r}\right)$, and that a proof can be given by applying the methods of this section to the general setup of [BC09, Theorem 1.5.6]. For example, when $A=\mathbb{F}$, such a statement can be deduced directly from [WE18b, Theorems 11.2.1 and 12.3.1].

In fact, a version for types $\left(d_{1}, d_{2}\right)$ can be deduced from Theorem 4.3 .5 by Morita equivalence, as in [BC09, Section 1.3.2]. 
Proof. We set $B=B^{\text {red }} \otimes_{R_{\bar{D}}^{\text {red }}} A$ and $B^{\mathcal{C}}=B^{\mathcal{C} \text {,red }} \otimes_{R_{\bar{D}}^{\mathcal{C}, \text { red }}} A$ to simplify notation. We consider the diagram

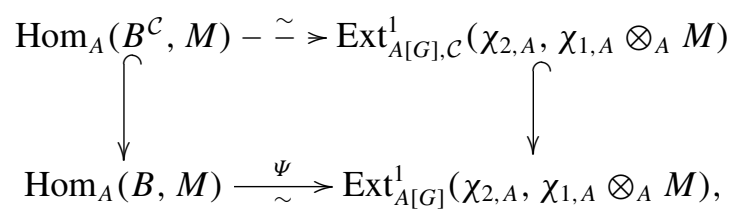

where $\Psi$ isomorphism of Proposition 4.2.7, and where the dotted arrow is the isomorphism we wish to construct. Since this diagram is canonically isomorphic to the one obtained by replacing $A$ by the image of $A \rightarrow \operatorname{End}_{A}(M)$, we can and do assume that $M$ is a faithful $A$-module. Given a class $c \in \operatorname{Ext}_{A[G]}^{1}\left(\chi_{2, A}, \chi_{1, A} \otimes_{A} M\right)$, we have to show that the map $f_{c}=\Psi^{-1}(c): B \rightarrow M$ factors through $B^{\mathcal{C}}$ if and only if $c \in \operatorname{Ext}_{A[G], \mathcal{C}}^{1}\left(\chi_{2, A}, \chi_{1, A} \otimes_{A} M\right)$.

Following the proof of Proposition 4.2.7, we see that the class $c$ has an extension module $E_{M} e_{2}$ where $E_{M}=\left(\begin{array}{cc}A & M \\ 0 & A\end{array}\right)$ is a GMA and $f_{c}$ induces a morphism of GMAs $E_{\bar{D}}^{\text {red }} \otimes_{R_{\bar{D}}^{\text {red }}} A \rightarrow E_{M}$ by

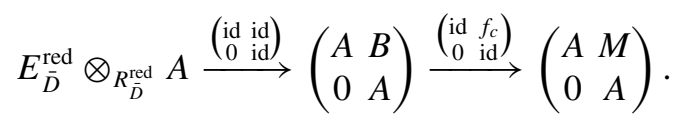

Since $M$ is a faithful $A$-module, we see that $E_{M} e_{2}$ is a faithful Cayley-Hamilton $G$-module with Cayley-Hamilton algebra $\left(E_{M}, D_{\mathcal{E}_{M}}\right)$. By Theorem 2.6.4, $E_{M} e_{2}$ has $\mathcal{C}$ if and only if $\left(E_{M}, D_{\mathcal{E}_{M}}\right)$ has $\mathcal{C}$ as a Cayley-Hamilton representation of $G$. By Lemmas 4.3.2, 2.4.5, and Theorem 2.5.3, we are reduced to showing that $f_{c}$ factors through $B^{\mathcal{C}}$ if and only if the map $E_{\bar{D}} \rightarrow E_{\bar{D}}^{\text {red }} \otimes_{R_{\bar{D}}^{\text {red }}} A \rightarrow E_{M}$ given by (4.3.7) factors through $E_{\bar{D}}^{\mathcal{C}}$.

If $f_{c}$ factors through $B^{\mathcal{C}}$, then we see that the map $E_{\bar{D}}^{\text {red }} \otimes_{R_{\bar{D}}^{\text {red }}} A \rightarrow E_{M}$ of (4.3.7) agrees with the map

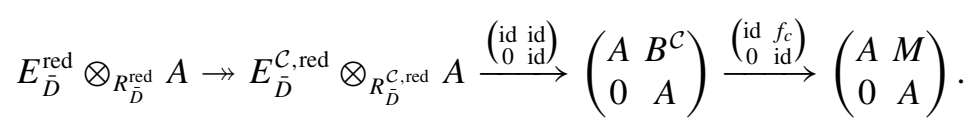

Hence the map $E_{\bar{D}} \rightarrow E_{M}$ factors though $E_{\bar{D}}^{\mathcal{C} \text {,red }}$, which is a quotient of $E_{\bar{D}}^{\mathcal{C}}$.

Conversely, suppose that $\left(E_{\bar{D}}, D_{E_{\bar{D}}}^{u}\right) \rightarrow\left(E_{M}, D_{\mathcal{E}_{M}}\right)$ factors through $\left(E_{\bar{D}}^{\mathcal{C}}, D_{E_{\bar{D}}^{\mathcal{C}}}\right)$. Since $\left(E_{M}, D_{\mathcal{E}_{M}}\right)$ is reducible, this implies that the map $E_{\bar{D}} \rightarrow E_{M}$ factors through a map $g: E_{\bar{D}}^{\mathcal{C} \text {,red }} \otimes_{R_{\bar{D}}^{\mathcal{C} \text {,red }}} A \rightarrow E_{M}$. By (4.3.7), we see that $f_{c}$ factors through $e_{1} g e_{2}: B^{\mathcal{C}} \rightarrow M$. 


\section{Examples}

The follow examples of conditions $\mathcal{C}$ could be useful in arithmetic applications.

5.1. Unramified local condition. Let $\ell$ be a prime number and let $K$ be a finite field extension of $\mathbb{Q}_{\ell}$. Let $G=\operatorname{Gal}(\bar{K} / K)$, which satisfies the $\Phi_{p}$ finiteness condition because it is topologically finitely generated. Let $H=I_{\ell} \subset G$ be the inertia subgroup. The condition $\operatorname{Mod}_{\mathbb{Z}_{p}[G / H]}^{\text {fin }} \subset \operatorname{Mod}_{\mathbb{Z}_{p}[G]}^{\text {in }}$, as in Example 2.3.2, is called unramified.

5.2. Local conditions at $\ell=p$. Retain the same notation as the previous subsection, but now assume $\ell=p$. Let $O_{K} \subset K$ denote the ring of integers. In this case, there are many conditions on representations of $G$ in $\mathbb{Q}_{p}$-vector spaces, coming from $p$-adic Hodge theory. Some of these conditions descend to $\operatorname{Mod}_{\mathbb{Z}_{p}[G]}^{\text {fin }}$ as follows.

DEFINITIONS 5.2.1. Let $V$ be an object of $\operatorname{Mod}_{\mathbb{Z}_{p}[G]}^{\text {fin }}$ and let $a \leqslant b$ be integers.

(1) We call $V$ finite flat if there is a finite-flat group scheme $\mathcal{G}$ over $O_{K}$ such that $V \cong \mathcal{G}(\bar{K})$ as $\mathbb{Z}_{p}[G]$-modules.

(2) We call an object $V \in \operatorname{Mod}_{\mathbb{Z}_{p}[G]}^{\mathrm{fin}}$ torsion crystalline (respectively semistable) with Hodge-Tate weights in $[a, b]$ if there is a crystalline (respectively semistable) representation $\rho: G \rightarrow \mathrm{GL}(W)$ with Hodge-Tate weights in $[a, b]$ and a $G$-stable $\mathbb{Z}_{p}$-lattice $T \subset W$ such that $V$ is isomorphic to a subquotient of $T$.

Let $\mathcal{C}_{\text {flat }} \subset \operatorname{Mod}_{\mathbb{Z}_{p}[G]}^{\text {fin }}$ denote the full subcategory of finite-flat objects. Ramakrishna has proven that $\mathcal{C}_{\text {flat }}$ is stable [Ram93, Section 2].

Let $\mathcal{C}_{\text {crys, }[a, b]}, \mathcal{C}_{\text {st },[a, b]} \subset \operatorname{Mod}_{\mathbb{Z}_{p}[G]}^{\text {fin }}$ denote the full subcategories that are torsion crystalline (respectively semistable) with Hodge-Tate weights in $[a, b]$. Since the category of crystalline (respectively semistable) representations with Hodge-Tate weights in $[a, b]$ is closed under finite direct sums, we see that $\mathcal{C}_{\text {crys, }[a, b]}$ and $\mathcal{C}_{\text {st, }[a, b]}$ are closed under finite direct sums. They are also closed under isomorphisms and subquotients by definition, so we see that they are stable.

REMARK 5.2.2. It is known that, for a $\mathbb{Z}_{p}[G]$-module $M$ that is finitely generated and free as a $\mathbb{Z}_{p}$-module, $M \otimes_{\mathbb{Z}_{p}} \mathbb{Q}_{p}$ is crystalline (respectively semistable) with Hodge-Tate weights in $[a, b]$ if and only if $M \otimes_{\mathbb{Z}_{p}} \mathbb{Z} / p^{n} \mathbb{Z}$ is torsion crystalline (respectively semistable) with Hodge-Tate weights in $[a, b]$ for all $n \geqslant 1$. This 
was conjectured by Fontaine, and proven by Liu using results of Kisin, following partial results of Ramakrishna, Berger, and Breuil. It is also known that there is an equivalence of categories $\mathcal{C}_{\text {flat }} \cong \mathcal{C}_{\text {crys,[0,1] }}$ for $p>2$. See [Liu07] and the references given there.

REMARK 5.2.3. If $\bar{D}$ is residually multiplicity-free and $\mathcal{C}$ is one of the conditions about, then a Zariski-closed substack $\widetilde{\operatorname{Rep}_{\bar{D}}} \subset \mathcal{} \subset \operatorname{Rep}_{\bar{D}}$ and a quotient $R_{\bar{D}} \rightarrow \tilde{R}_{\bar{D}}^{\mathcal{C}}$ were constructed in [WE18a, Section 7.1]. The two constructions will have the same generic fiber (over $\mathbb{Z}_{p}$ ), as this is where the construction of crystalline loci is done in [WE18a]. That is, $\tilde{R}_{\bar{D}}^{\mathcal{C}}[1 / p] \cong R_{\bar{D}}^{\mathcal{C}}[1 / p]$, and similarly for $\widetilde{\operatorname{Rep}}_{\bar{D}}^{\mathcal{C}}$ and $\operatorname{Rep}_{\bar{D}}^{\mathcal{C}}$. In particular, the geometric properties of the generic fibers proved in [WE18a, Proposition 6.4.4 and Corollary 7.1.5] apply to the generic fibers of the $\operatorname{Rep}_{\bar{D}}^{\mathcal{C}}$ and $R_{\bar{D}}^{\mathcal{C}}$ constructed in this paper.

When the $\mathbb{Z}_{p}$-integral structures differ, we believe that the constructions in this paper will be better behaved: here, we make natively integral constructions, while the integral model of [WE18a] is made by flat closure relative to $\mathbb{Z}_{p}$.

5.3. Global conditions. Let $F$ be a number field with algebraic closure $\bar{F}$ and let $S$ be a finite set of places of $F$. Let $G=\operatorname{Gal}\left(F_{S} / F\right)$, where $F_{S} \subset \bar{F}$ is the maximal extension of $F$ unramified outside $S$. Then $G$ satisfies $\Phi_{p}$ by class field theory.

For each $v \in S$, choose a decomposition group $G_{v} \subset G$ (so $G_{v}$ is of the type considered in the previous subsections) and a stable condition $\mathcal{C}_{v} \subset \operatorname{Mod}_{\mathbb{Z}_{p}\left[G_{v}\right]}^{\text {fin }}$. Then there is a corresponding stable condition $\mathcal{C} \subset \operatorname{Mod}_{\mathbb{Z}_{p}[G]}^{\text {fin }}$, as in Example 2.3.3. The Selmer groups of Example 4.3.3 correspond to such a $\mathcal{C}$.

\section{Acknowledgements}

We thank Kȩstutis Česnavičius for pointing out an unusual discrepancy about the numbering in [Che14]. We thank James Newton for helpful comments and discussions about applications to modularity lifting and the paper $\left[\mathrm{ACC}^{+} \mathbf{1 8}\right.$ ], which led us to include Corollary 3.3.2. We thank the anonymous referees for many helpful comments that have led to improvements in the exposition. The intellectual debt owed to the works of Bellaïche-Chenevier [BC09] and Chenevier [Che14] will be clear to the reader. We thank Joël Bellaïche for his interest in and encouragement about this work. P.W. was supported by the National Science Foundation under the Mathematical Sciences Postdoctoral Research Fellowship No. 1606255 and Grant No. DMS-1638352. C.W.E. was 
supported by the Engineering and Physical Sciences Research Council grant EP/L025485/1.

\section{References}

[ACC $\left.{ }^{+} 18\right]$ P. B. Allen, F. Calegari, A. Caraiani, T. Gee, D. Helm, B. V. Le Hung, J. Newton, P. Scholze, R. Taylor and J. A. Thorne, 'Potential automorphy over CM fields', Preprint, 2018, arXiv:1812.09999v1 [math.NT].

[Ami80] S. A. Amitsur, 'On the characteristic polynomial of a sum of matrices', Linear Multilinear Algebra 8(3) (1979/80), 177-182.

[BC09] J. Bellaïche and G. Chenevier, 'Families of Galois representations and Selmer groups', Astérisque 324 (2009), xii+314.

[CS16] F. Calegari and J. Specter, 'Pseudo-representations of weight one'. Undated preprint. Accessed January 17, 2019 at http://www.math.jhu.edu/ jspecter/Pseudo.pdf. First version of [CS19], circa 2016.

[CS19] F. Calegari and J. Specter, 'Pseudo-representations of weight one are unramified', Algebra Number Theory (2019) Preprint, arXiv:1906.10473v1 [math.NT], to appear.

[Che14] G. Chenevier, 'The $p$-adic analytic space of pseudocharacters of a profinite group, and pseudorepresentations over arbitrary rings', in Automorphic Forms and Galois Representations: Vol. I, London Mathematical Society Lecture Note Series, 414 (Cambridge University Press, Cambridge, 2014), 221-285. We follow the numbering of the online version arXiv:0809.0415v2, which differs from the print version.

[Gro60] A. Grothendieck, 'Éléments de géométrie algébrique. I. Le langage des schémas', Publ. Math. Inst. Hautes Études Sci. 4 (1960), 5-228.

[Liu07] T. Liu, 'Torsion $p$-adic Galois representations and a conjecture of Fontaine', Ann. Sci. Éc. Norm. Supér. (4) 40(4) (2007), 633-674.

[Maz77] B. Mazur, 'Modular curves and the Eisenstein ideal', Publ. Math. Inst. Hautes Études Sci. 47 (1977), 33-186.

[Maz89] B. Mazur, 'Deforming Galois representations', in Galois Groups Over Q (Berkeley, CA, 1987), Math. Sci. Res. Inst. Publ., 16 (Springer, New York, 1989), 385-437.

[Nys96] L. Nyssen, 'Pseudo-représentations', Math. Ann. 306(2) (1996), 257-283.

[Pro87] C. Procesi, 'A formal inverse to the Cayley-Hamilton theorem', J. Algebra 107(1) (1987), 63-74.

[Ram93] R. Ramakrishna, 'On a variation of Mazur's deformation functor', Compos. Math. 87(3) (1993), 269-286.

[Rou96] R. Rouquier, 'Caractérisation des caractères et pseudo-caractères', J. Algebra 180(2) (1996), 571-586.

[Tay91] R. Taylor, 'Galois representations associated to Siegel modular forms of low weight', Duke Math. J. 63(2) (1991), 281-332.

[WE18a] C. Wang-Erickson, 'Algebraic families of Galois representations and potentially semistable pseudodeformation rings', Math. Ann. 371(3-4) (2018), 1615-1681.

[WE18b] C. Wang-Erickson, 'Deformations of residually reducible Galois representations via $A_{\infty}$-algebra structure on Galois cohomology', Preprint, 2018, arXiv:1809.02484v1 [math.NT].

[Wil88] A. Wiles, 'On ordinary $\lambda$-adic representations associated to modular forms', Invent. Math. 94(3) (1988), 529-573. 
[WWE17a] P. Wake and C. Wang-Erickson, 'Ordinary pseudorepresentations and modular forms', Proc. Amer. Math. Soc. B 4 (2017), 53-71.

[WWE17b] P. Wake and C. Wang-Erickson, 'The rank of Mazur's Eisenstein ideal', Duke Math. J., to appear. Preprint, 2017, arXiv:1707.01894v2 [math.NT].

[WWE18a] P. Wake and C. Wang-Erickson, 'Pseudo-modularity and Iwasawa theory', Amer. J. Math. 140(4) (2018), 977-1040.

[WWE18b] P. Wake and C. Wang-Erickson, 'The Eisenstein ideal with squarefree level', Preprint, 2018, arXiv:1804.06400v2 [math.NT]. 